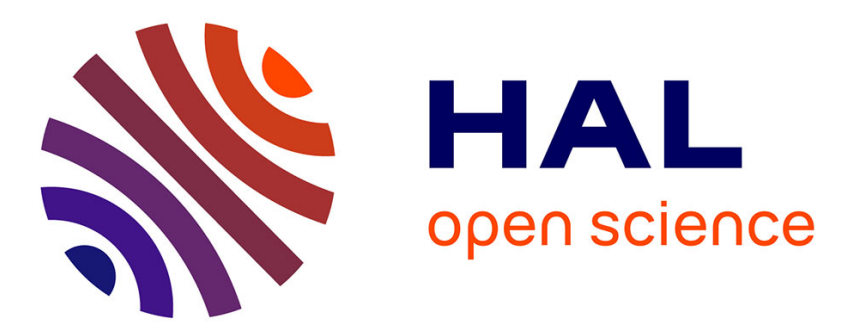

\title{
La mobilisation des bois en provenance des forêts soumises au régime forestier en 1984 et les prix obtenus aux grandes ventes de l'automne 1984 - Calendrier des grandes ventes d'automne \\ - [.]office National Des Forets Paris
}

\section{To cite this version:}

- [.]office National Des Forets Paris. La mobilisation des bois en provenance des forêts soumises au régime forestier en 1984 et les prix obtenus aux grandes ventes de l'automne 1984 - Calendrier des grandes ventes d'automne. Revue forestière française, 1985, 37 (4), pp.262-280. 10.4267/2042/21808 . hal-03535002

\section{HAL Id: hal-03535002 \\ https://hal.science/hal-03535002}

Submitted on 19 Jan 2022

HAL is a multi-disciplinary open access archive for the deposit and dissemination of scientific research documents, whether they are published or not. The documents may come from teaching and research institutions in France or abroad, or from public or private research centers.
L'archive ouverte pluridisciplinaire HAL, est destinée au dépôt et à la diffusion de documents scientifiques de niveau recherche, publiés ou non, émanant des établissements d'enseignement et de recherche français ou étrangers, des laboratoires publics ou privés. 


\title{
LA MOBILISATION DES BOIS EN PROVENANCE DES FORÊTS SOUMISES AU RÉGIME FORESTIER EN 1984 ET LES PRIX OBTENUS AUX GRANDES VENTES DE L'AUTOMNE 1984
}

\author{
OFFICE NATIONAL DES FORÊTS
}

En 1984, la situation du marché du bois aura été variable en fonction des secteurs: favorable en ce qui concerne le Chêne sous l'action d'une demande étrangère soutenue, plus incertaine en matière de Hêtre, avec l'afflux de chablis dans ces essences en 1984 (en Picardie, Normandie, Champagne en février; dans les Vosges en juillet; en Normandie et en Picardie à nouveau, en novembre), assez morose en matière de résineux pour lesquels le marché intérieur se révélait quelque peu saturé suite aux chablis du Massif Central et malgré un marché international plutôt ferme.

Les résultats des ventes de l'automne 1984 s'inscrivent bien dans ce contexte, puisqu'ils traduisent, par rapport à l'automne $1983^{(1)}$, des invendus plus importants en résineux accompagnés d'une baisse de leur prix, une demande soutenue en Chêne entraînant une hausse sensible des bois de qualité, une tendance un peu irrégulière sur le Hêtre.

Pour la troisième année consécutive, les quantités mobilisées de bois en provenance des forêts soumises au régime forestier ont atteint un niveau exceptionnel:

- 1982: $11300000 \mathrm{~m}^{3}$ contre $10500000 \mathrm{~m}^{3}$ en année moyenne,

- 1983: $12650000 \mathrm{~m}^{3}$

$-1984: 13450000 \mathrm{~m}^{3}$

Ces niveaux élevés sont liés à l'abondance des chablis issus de tempêtes successives (cf. supra) et des bois dépérissants (attaques de Scolytes, Cryptococcus...).

Ces bois ont été mobilisés à concurrence de $65 \%$ vendus en bloc et sur pied, $14 \%$ vendus façonnés (Alsace et Moselle), 3,5\% vendus à l'unité de produits et $17,5 \%$ délivrés aux collectivités propriétaires pour leurs propres besoins.

(1) Voir l'article semblable à celui-ci, concernant les grandes ventes 1983, et paru dans le $\mathrm{n}^{\circ}$ 3/1984 de cette revue, pp. 181-200. 


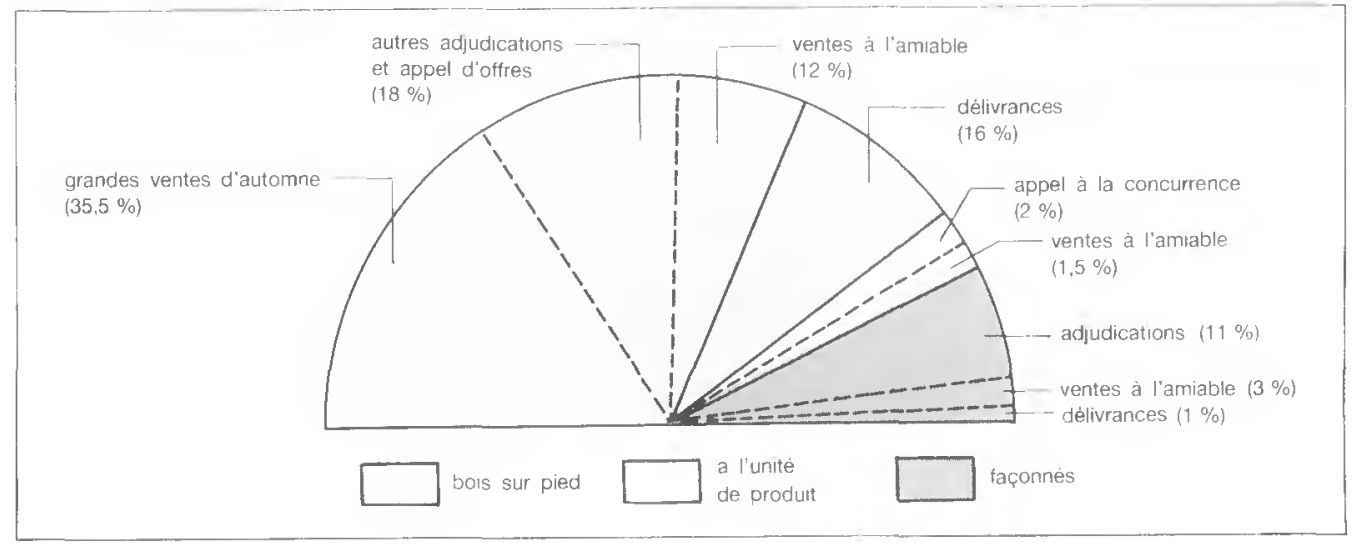

Tableau I

La mobilisation de bois en forêt soumise en 1984

\begin{tabular}{|c|c|c|c|c|c|c|}
\hline \multirow[t]{2}{*}{ (En millions de $\mathrm{m}^{3}$ ) } & \multirow{2}{*}{$\begin{array}{c}\text { Forêts } \\
\text { domaniales }\end{array}$} & \multicolumn{2}{|c|}{$\begin{array}{c}\text { Forêts des collectivités } \\
\text { publiques }\end{array}$} & \multicolumn{3}{|c|}{$\begin{array}{l}\text { Toutes forêts soumises } \\
\text { au régime forestier }\end{array}$} \\
\hline & & ventes & délivrances & 1984 & 1983 & 1982 \\
\hline $\begin{aligned} 1 \text { - Bois sur pied : } \\
\\
\text { - Feuillus ......... } \\
\text { - Résineux . . . . . . } \\
\text { - Taillis et houppiers }\end{aligned}$ & $\begin{array}{l}2,1 \\
1,8 \\
1,2\end{array}$ & $\begin{array}{l}1,0 \\
2,3 \\
0,9\end{array}$ & $\begin{array}{l}0,7 \\
0,4 \\
1,1\end{array}$ & $\begin{array}{l}3,80 \\
4,50 \\
3,20\end{array}$ & $\begin{array}{l}3,45 \\
4,50 \\
2,80\end{array}$ & $\begin{array}{l}3,20 \\
3,55 \\
2,70\end{array}$ \\
\hline Total $1 \ldots \ldots \ldots \ldots$ & 5,1 & 4,2 & 2,2 & 11,50 & 10,75 & 9,45 \\
\hline 2 - Bois façonnés ...... & 0,9 & 1,0 & 0,1 & 2,00 & 1,90 & 1,85 \\
\hline TOTAL $1+2$ & 6,0 & 5,2 & 2,3 & 13,50 & 12,65 & 11,30 \\
\hline
\end{tabular}

En se chiffrant à $14800000 \mathrm{~m}^{3}$, l'offre globale 1984 en provenance de la forêt soumise (sur pied + à l'unité de produit et dénombrés + façonnés; bois nouvellement mis en vente + remis en vente + délivrés) aura finalement dépassé celle de 1983, pourtant déjà particulièrement élevée (cf. tableau en annexe IV). Il faut y voir ici le cumul des effets cités plus haut.

Cependant, le stock d'invendus en_fin d'année - $1370000 \mathrm{~m}^{3}$-, tout en étant un peu plus important que celui constaté au 31 décembre 1983, reste inférieur à celui de 1981 et 1982 (cf. tableau en annexe IV).

Toutes les essences ont été concernées par cette mobilisation accrue, y compris le Pin maritime et les Résineux divers pourtant peu touchés par les diverses tornades. II est vrai que pour ces essences, la mobilisation en a toujours été irrégulière dans le temps (voir diagrammes les concernant dans la suite de l'article).

En fin de compte, en 1984, malgré la baisse des prix de nombreuses essences (soit en raison de la conjoncture, soit en raison d'une "décote-chablis"), les recettes tant domaniales que des collectivités publiques ont progressé très légèrement par rapport à 1983, en raison de l'augmentation des volumes mobilisés et de la hausse des prix du Chêne. 
OFFICE NATIONAL DES FORÊTS

Tableau II

Recettes-Bois

\begin{tabular}{|c|c|c|c|c|c|c|c|c|}
\hline \multirow{2}{*}{ (En millions de $F$ ) } & \multicolumn{4}{|c|}{ Forêts domaniales } & \multicolumn{4}{|c|}{ Forêts des collectivités } \\
\hline & 1981 & 1982 & 1983 & 1984 & 1981 & 1982 & 1983 & 1984 \\
\hline $\begin{array}{l}\text { Bois sur pied: } \\
\text { - Ventes d'automne ....... } \\
\text { - Autres ventes sur pied .... }\end{array}$ & $\begin{array}{l}511,0 \\
140,6\end{array}$ & $\begin{array}{l}511,6 \\
157,3\end{array}$ & $\begin{array}{l}527,5 \\
322,0\end{array}$ & $\begin{array}{l}550,4 \\
302,5\end{array}$ & $\begin{array}{l}403,7 \\
188,6\end{array}$ & $\begin{array}{l}425,8 \\
192,1\end{array}$ & $\begin{array}{l}427,3 \\
261,5\end{array}$ & $\begin{array}{l}412,8 \\
339,9\end{array}$ \\
\hline$\ldots \ldots \ldots \ldots$ & 651,6 & 68.9 & 49,5 & 52,9 & 592,3 & 617,9 & 688,8 & 752,7 \\
\hline $\begin{array}{l}\text { - Estimation-valeur des déli- } \\
\text { vrances ............. } \\
\text { Bois façonnés: } \\
\text { - Ventes .............. } \\
\text { - Estimation-valeur des déli- } \\
\text { vrances ............. }\end{array}$ & $\begin{array}{r}210,3 \\
5,3\end{array}$ & $\begin{array}{r}198,9 \\
5,4\end{array}$ & $\begin{array}{r}225,1 \\
5,6\end{array}$ & $\begin{array}{r}245,4 \\
6,0\end{array}$ & $\begin{array}{r}273,6 \\
10,2\end{array}$ & $\begin{array}{r}254,6 \\
12,7\end{array}$ & $\begin{array}{r}280,1 \\
13,9\end{array}$ & $\begin{array}{r}289,7 \\
13,1\end{array}$ \\
\hline TOTAL GÉNÉRAL & 867,3 & 873,4 & 1080,3 & 1104,7 & 1034,0 & 1053,2 & 1151,9 & 1222,3 \\
\hline
\end{tabular}

\section{LE CHÊNE}

Pour la deuxième année consécutive, les quantités de Chêne mobilisées en forêt soumise auront été importantes en raison des nombreux volumes de cette essence abattus par les tempêtes (en novembre 1982, dans l'Allier et l'Indre et en juillet 1984, dans les Vosges). Ces bois ont aisément trouvé preneurs, à des prix plutôt en hausse; en effet, le Chêne a èté très recherché cette année sous l'action d'une forte demande étrangère, comme en témoigne l'augmentation sensible de nos exportations tant en grumes qu'en sciages en 1984 (de l'ordre de $30 \%$ en volume).

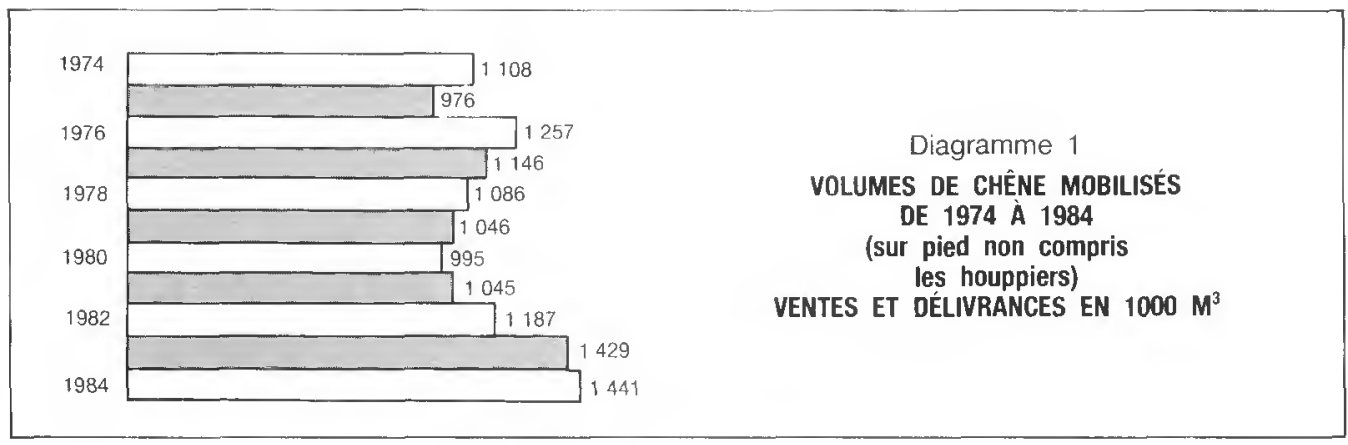

A l'automne, le prix du Chêne sur pied a enregistré une hausse moyenne de 16,5\%, marquant en fait une hausse plus forte sur les bois de belle qualité et une quasi-stabilité des bois de qualité courante. L'origine du mouvement de hausse des produits de qualité est claire: la hausse très rapide du dollar par rapport à toutes les monnaies européennes a fortement pénalisé depuis 2 ans les ventes de Chêne d'Amérique sur les marchés européens, et notamment en Allemagne: ce handicap, imposé à notre principal concurrent sur ces marchés, a permis une reprise importante, en volume et en valeur, des ventes de Chêne français à l'exportation, et donc sur le marché intérieur. 
II convient cependant de noter que malgré ces hausses, les prix actuels du Chêne en francs courants sont sensiblement au même niveau qu'en 1978, ce qui traduit une baisse proche de $50 \%$ en francs constants.

L'analyse des prix par catégorie de diamètre dans les départements les plus producteurs confirme bien la tendance relevée au plan national; pour les gros diamètres. les invendus sont réduits et les hausses oscillent entre 13 et $30 \%$; pour les moyens diamètres, les prix fluctuerit autour des prix de l'an passé, selon la qualite des bois et l'importance des invendus.

Graphique 2

\section{CHÊNE}

ÉVOLUTION

DES PRIX MOYENS

AUX GRANDES VENTES

(taxe forfaitaire

comprise)

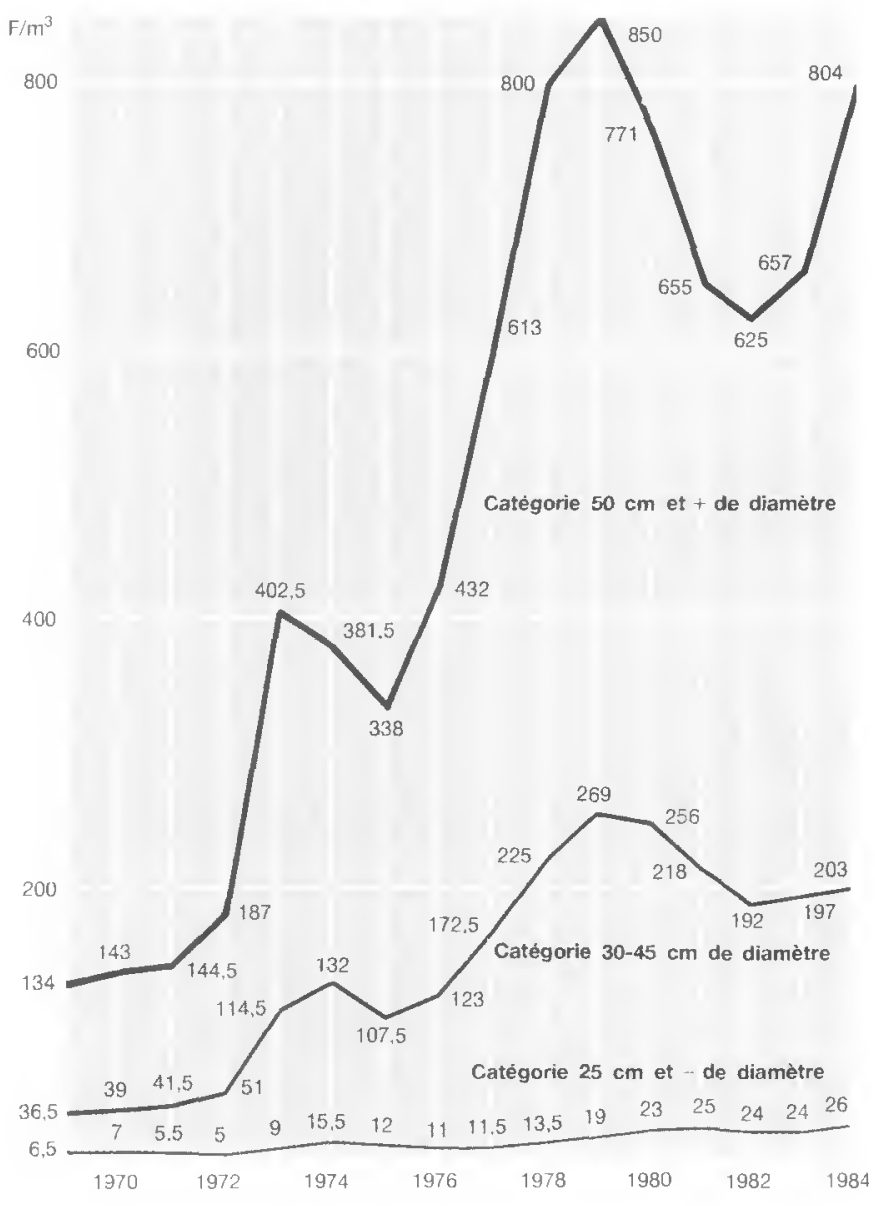

Tableau III

Chêne de $50 \mathrm{~cm}$ et plus de diamètre

Principaux départements producteurs - Grandes ventes 1984

\begin{tabular}{|c|c|c|c|c|}
\hline Départements & $\begin{array}{c}\text { Prix moyen } \\
1984\end{array}$ & $\begin{array}{l}\text { Variation (1) } \\
1984-1983\end{array}$ & $\begin{array}{l}\text { Volume vendu } \\
\qquad\left(\mathrm{m}^{3}\right)\end{array}$ & $\begin{array}{l}\text { Proportion } \\
\text { d'invendus }\end{array}$ \\
\hline Allier & 1070 & $+30 \%$ & 12800 & $0,5 \%$ \\
\hline Loir-et-Cher & 1158 & $+25 \%$ & 12750 & $2 \%$ \\
\hline Indre $\ldots \ldots \ldots \ldots$ & 1168 & $+2 \%$ & 18360 & $5 \%$ \\
\hline Orne ... & 834 & $+18 \%$ & 14870 & $5 \%$ \\
\hline Nièvre... & 938 & $+13 \%$ & 19670 & $13 \%$ \\
\hline Côte-d'Or & 817 & $+13 \%$ & 33380 & $9 \%$ \\
\hline Haute-Marne .... . & 814 & $+13 \%$ & 25270 & $11 \%$ \\
\hline
\end{tabular}

(1) Départements fortement touches par la tempète de novembre 1982. Des quantites importantes de chêne (qualıté tranchage en particulier) ont eté commercialisées au printemps 83. 
OFFICE NATIONAL DES FORÊTS

Tableau IV

Chêne de 30 à $45 \mathrm{~cm}$ de diamètre

Principaux départements producteurs - Grandes ventes 1984

\begin{tabular}{|c|c|c|c|c|}
\hline Dèpartements & $\begin{array}{c}\text { Prix moyen } \\
1984\end{array}$ & $\begin{array}{l}\text { Variation }^{(1)} \\
1984-1983\end{array}$ & $\begin{array}{l}\text { Volume vendu } \\
\qquad\left(\mathrm{m}^{3}\right)\end{array}$ & $\begin{array}{l}\text { Proportion } \\
\text { d'invendus }\end{array}$ \\
\hline Allier (1) .. & 232 & $-18 \%$ & 12050 & $2 \%$ \\
\hline Loir-et-Cher . . & 196 & $-9 \%$ & 15600 & $2 \%$ \\
\hline Indre .... & 185 & $-3 \%$ & 12670 & $24 \%$ \\
\hline Orne .. & 249 & $+2 \%$ & 13690 & $4 \%$ \\
\hline Nièvre... & 110 & - & 12630 & $20 \%$ \\
\hline Côte-d'Or .... & 195 & $+16 \%$ & 19130 & $18 \%$ \\
\hline Haute-Marne ... & 174 & $+7 \%$ & 15100 & $19 \%$ \\
\hline
\end{tabular}

(1) Variation peu significative en raison de l'abondance des chablis commercialises hors automne 83 .

\section{LE HÊTRE}

Depuis trois ans, l'offre de Hêtre atteint un niveau élevé ; en effet, les ravages que causent en Normandie le Cryptococcus ont entraîné un afflux de Hêtre sur le marché dans cette région; de plus, en 1984, les tempêtes successives de février, juillet et novembre ont provoqué d'abondants chablis en cette essence dans la moitie Nord de la France (environ $1750000 \mathrm{~m}^{3}$ ).

L'activité soutenue de la demande étrangère (forte augmentation des exportations de grumes de Hêtre en 1984) a facilité l'ècoulement de ces quantités accrues.

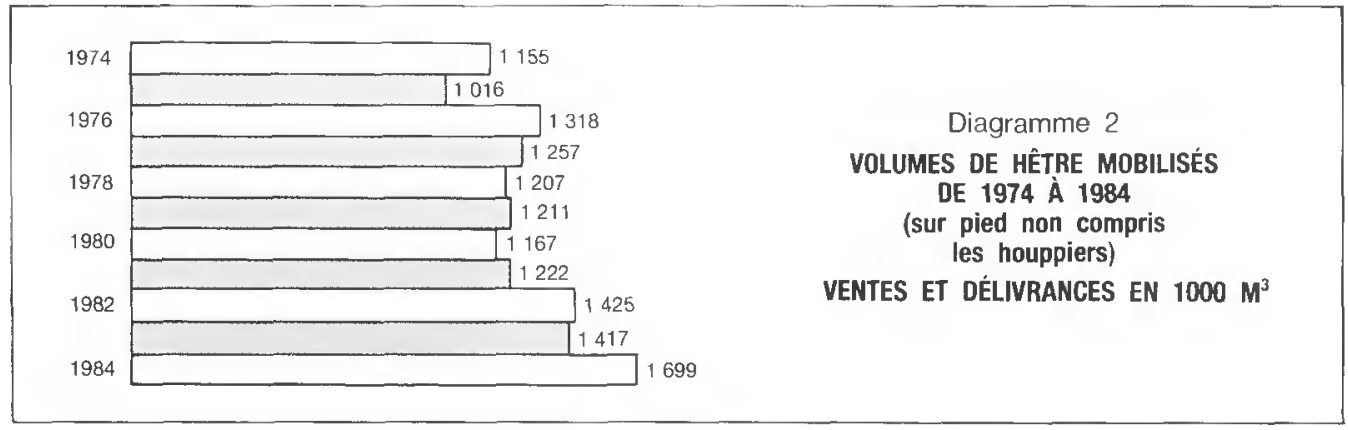

C'est donc dans un contexte perturbé que se sont déroulées les ventes d'automne: les chablis des Vosges, intervenus en juillet, ont conduit l'Office à modifier son offre dans les régions proches de la zone sinistrée, pour faciliter l'écoulement des chablis.

Le prix moyen du Hêtre qui se dégage n'est donc pas très significatif, dans la mesure où les quantités et qualités offertes en Hêtre à l'automne 84 ne sont pas vraiment comparables à celles de l'automne 83.

La baisse moyenne de $17 \%$ n'est pas réelle à qualité égale. On le voit en étudiant les résultats dans un département èpargné (mais cependant proche de la zone sinistrẻe) comme la Meuse où la baisse du prix moyen du Hêtre de $40 \mathrm{~cm}$ et plus de diamètre n'est que de $3 \%$ (cf. tableau V). 
Les autres départements mentionnés, à l'exception des Pyrénées-Atlantiques, ont tous été touchés par le phénomène chablis. En fait, il semble que les prix du Hêtre de bonne qualitè se soient à peu près maintenus, alors que ceux des qualités moyennes ont baissé davantage.

Dans les Pyrénées-Atlantiques, l'importance des invendus empêche toute comparaison valable ; la demande a été faible, portant uniquement sur les coupes de vidange aisée; par alleurs, certains acheteurs habituels s'étaient approvisionnés en chablis de Normandie et Picardie.

Graphique 3

HÊTRE

ÉVOLUTION DES PRIX MOYENS

AUX GRANDES VENTES

(taxe forfaitaire comprise)

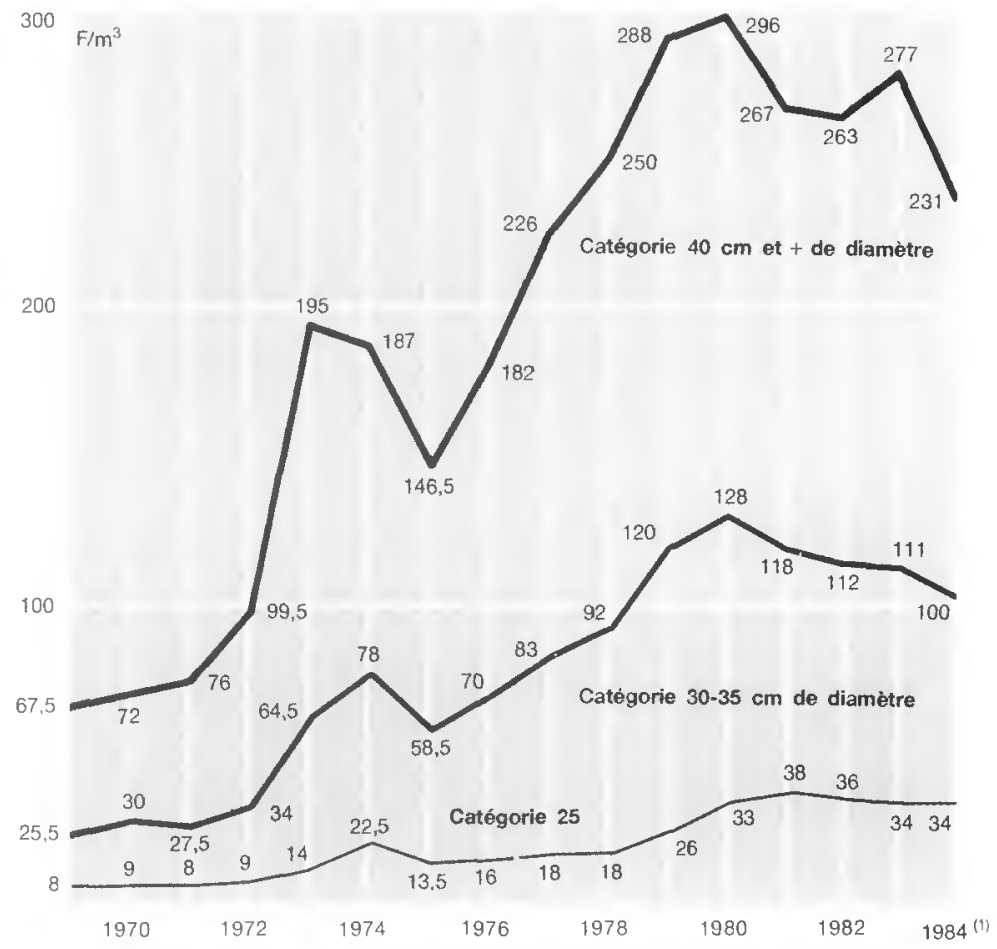

(1) 1984 : Prix peu significatifs en raison de l'abondance des chablıs de Hêtre en 1984 (Normandie-Picardie-Vosges) et de la réduction de l'offre d'automne dans ces régions.

Tableau V

Hêtre de $40 \mathrm{~cm}$ et plus de diamètre

Principaux départements producteurs - Grandes ventes 1984

\begin{tabular}{|c|c|c|c|c|}
\hline Départements & $\begin{array}{c}\text { Prix moyen } \\
1984\end{array}$ & $\begin{array}{l}\text { Variation } \\
1984-1983\end{array}$ & $\begin{array}{l}\text { Volume vendu } \\
\qquad\left(\mathrm{m}^{3}\right)\end{array}$ & $\begin{array}{l}\text { Proportion } \\
\text { d'invendus }\end{array}$ \\
\hline Eure/Seine-Maritime & 174 & $-36 \%$ & 77690 & $8 \%$ \\
\hline Oise .. & 153 & $-32 \%$ & 62200 & $7 \%$ \\
\hline Aisne . . & 146 & $-42 \%$ & 17740 & $2 \%$ \\
\hline Haute-Marne ...... & 336 & $-7 \%$ & 29040 & $18 \%$ \\
\hline Meuse $\ldots . .$. . & 253 & $-3 \%$ & 36900 & $27 \%$ \\
\hline Pyrénées-Atlantiques & 276 & $-23 \%$ & 11151 & $68 \%$ \\
\hline
\end{tabular}

(1) Variation peu significative en raison de l'abondance des chablis commercialisés hors automne 1983.

\section{LES AUTRES FEUILLUS}

Les volumes récoltés ont èté également en augmentation; en effet, les tempêtes de février, juillet et novembre 1984 ont touché essentiellement les forêts de feuillus; il y avait également, en mélange avec le Chêne ou le Hêtre, des feuillus divers. 
Comme d'habitude, le Merisier, l'Érable et le Frêne ont été recherchés, comme les belles qualités de Chêne.

Par ailleurs, la demande en bois de trituration ayant été active, les feuillus moins " nobles " se sont écoulés aisément.

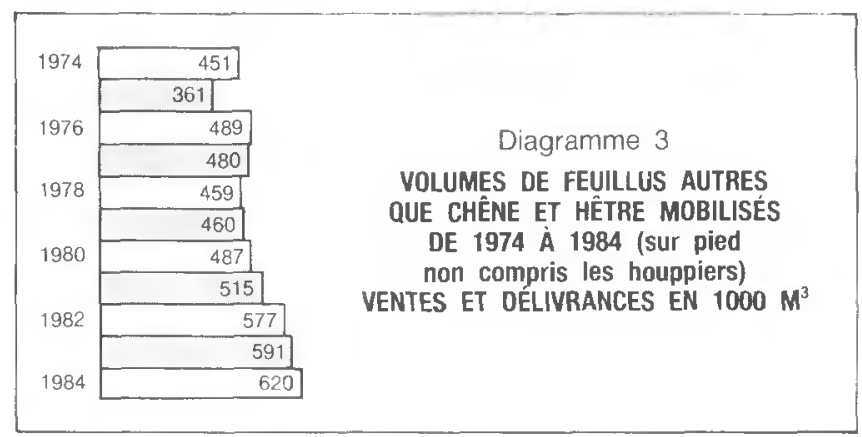

\section{LE SAPIN ET L'ÉPICÉA}

Les séquelles de la tempête de novembre 1982 se sont encore faits sentir en 1984 puisque d'importantes quantités de Sapin et d'Épicéa vendues à l'unité de produit en 1983 n'ont été exploitées et dénombrées qu'en 1984. La mobilisation de Sapin et d'Épicéa a donc encore été très forte en 1984, même si elle n'atteint pas le niveau de 1983 (cf. diagramme ci-après).

Malgré un marché international ferme, les cours du Sapin-Épicéa ont régressé, dans tous les massifs forestiers concernés, d'une façon assez homogene, d'environ $10 \%$ en moyenne (cf. graphique 4); cette baisse moyenne recouvre toutefois une quasi-stabilité des prix des belles coupes et une baisse nettement plus accentuée des prix des bois de qualité secondaire, conséquence probable de la saturation du marché des bois de coffrage, en raison d'une part des effets des chablis du Massif Central ef d'autre part des difficultés des travaux publics et du bâtiment.

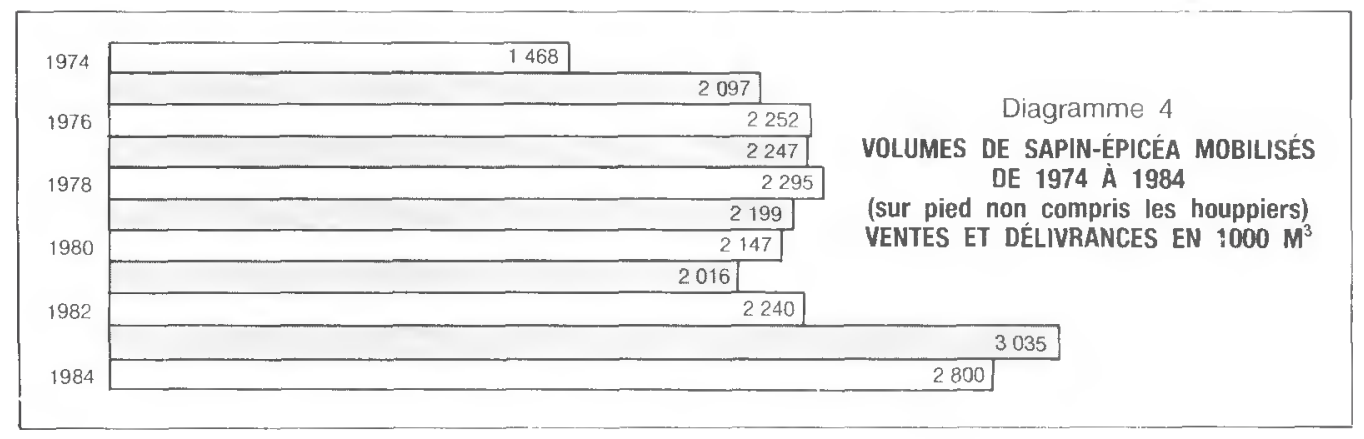

La courbe des prix des coupes d'amélioration de la vente de Pontarlier confirme bien cette analyse (cf. graphique 5).

A l'automne, les invendus ont d'ailleurs été relativement volumineux dans l'ensemble (28\%).

Les résultats des ventes dans les départements producteurs de Sapin et d'Épicéa font apparaître une baisse des prix quasi-générale. De plus, si les invendus restent plutôt modérés dans le quart Nord-Est de la France, ils sont au contraire très élevés dans les départements alpins et pyrénéens, ce qui fausse souvent les comparaisons de prix (en particulier en Savoie, d'autant plus qu'il s'agissait d'un département sinistré par la tempête de 1982 et dont le prix moyen 1983 reflétait ce sinistre). 

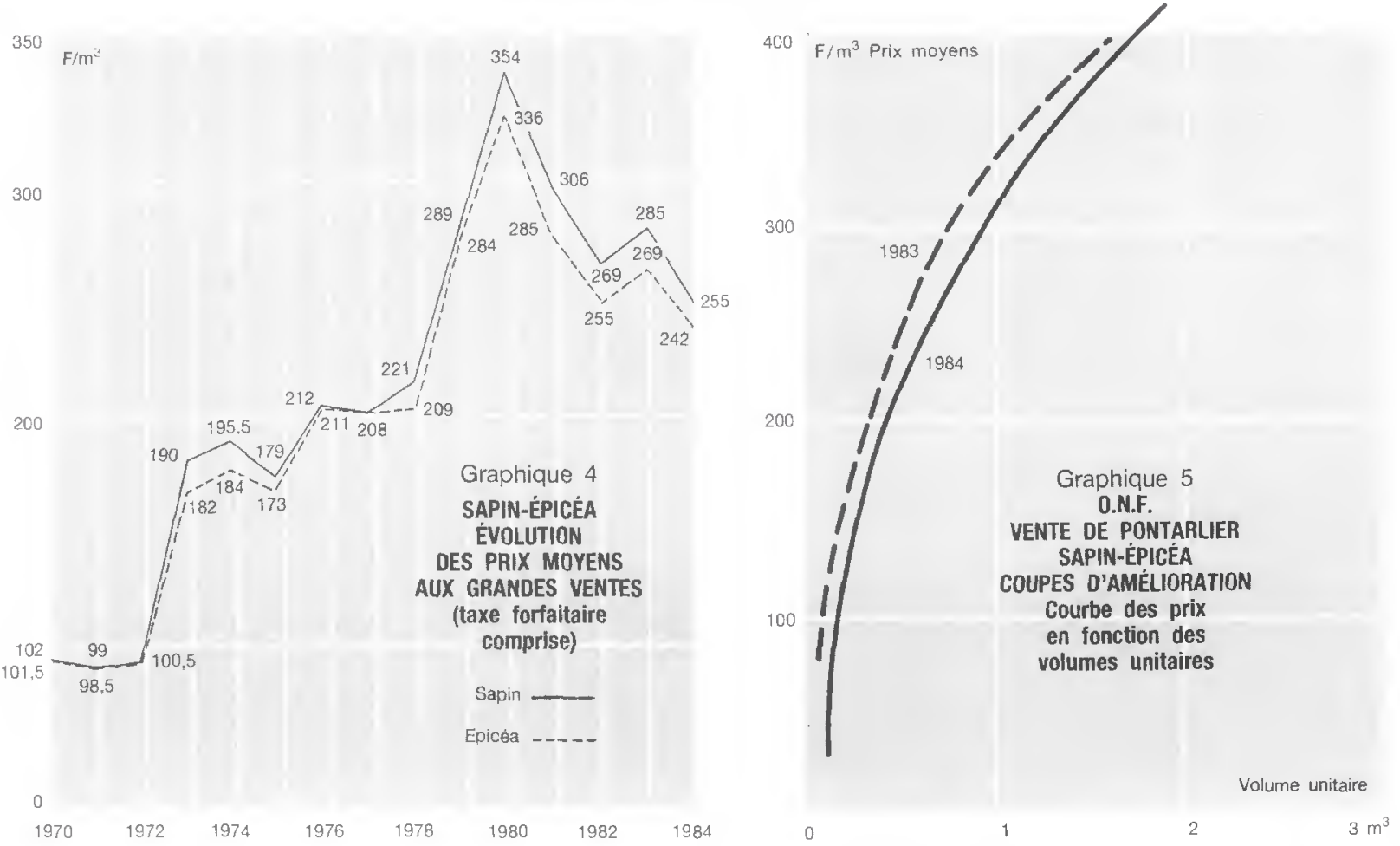

Tableau VI

Sapin et Épicéa de plus de $25 \mathrm{~cm}$ de diamètre

Principaux départements producteurs - Grandes ventes 1984

\begin{tabular}{|c|c|c|c|c|c|}
\hline Départements & Essences & $\begin{array}{c}\text { Prix moyen } \\
1984\end{array}$ & $\begin{array}{c}\text { Variation } \\
1984-1983\end{array}$ & $\begin{array}{c}\text { Volume } \\
\text { vendu }\left(\mathrm{m}^{3}\right)\end{array}$ & $\begin{array}{l}\text { Proportion } \\
\text { d'invendus }\end{array}$ \\
\hline Vosges ....... & $\begin{array}{l}\text { Sapin } \\
\text { Epicéa }\end{array}$ & $\begin{array}{l}280 \\
299\end{array}$ & $\begin{array}{l}-\quad 9 \% \\
-\quad 8 \%\end{array}$ & $\begin{array}{r}194100 \\
45960\end{array}$ & $\begin{array}{l}19 \% \\
15 \%\end{array}$ \\
\hline Doubs ......... & $\begin{array}{l}\text { Sapin } \\
\text { Epicéa }\end{array}$ & $\begin{array}{l}324 \\
360\end{array}$ & $\begin{array}{l}-14 \% \\
-13 \%\end{array}$ & $\begin{array}{l}78620 \\
62470\end{array}$ & $\begin{array}{r}13 \% \\
9 \%\end{array}$ \\
\hline Jura & $\begin{array}{l}\text { Sapin } \\
\text { Epicéa }\end{array}$ & $\begin{array}{l}300 \\
323\end{array}$ & $\begin{array}{r}-11 \% \\
-\quad 7 \%\end{array}$ & $\begin{array}{l}48720 \\
22220\end{array}$ & $\begin{array}{l}16 \% \\
15 \%\end{array}$ \\
\hline Ain $\ldots . . . .$. & $\begin{array}{l}\text { Sapin } \\
\text { Epicéa }\end{array}$ & $\begin{array}{l}260 \\
272\end{array}$ & $\begin{array}{l}-10 \% \\
-10 \%\end{array}$ & $\begin{array}{l}67450 \\
16780\end{array}$ & $\begin{array}{l}15 \% \\
13 \%\end{array}$ \\
\hline Haute-Savoie ............. & $\begin{array}{l}\text { Sapin } \\
\text { Epicéa }\end{array}$ & $\begin{array}{l}231 \\
235\end{array}$ & $\begin{array}{l}-10 \% \\
-\quad 9 \%\end{array}$ & $\begin{array}{r}6800 \\
26550\end{array}$ & $\begin{array}{l}67 \% \\
52 \%\end{array}$ \\
\hline Savoie ${ }^{11\}}$ & $\begin{array}{l}\text { Sapin } \\
\text { Epicéa }\end{array}$ & $\begin{array}{l}227 \\
215\end{array}$ & $\begin{array}{l}+94 \% \\
+17 \%\end{array}$ & $\begin{array}{l}10950 \\
17740\end{array}$ & $\begin{array}{l}62 \% \\
64 \%\end{array}$ \\
\hline Aude ${ }^{11}, \ldots \ldots \ldots \ldots \ldots \ldots$ & Sapin & 279 & $+6 \%$ & 22580 & $16 \%$ \\
\hline Hautes-Pyrénées .. & Sapin & 220 & $-2 \%$ & 25350 & $37 \%$ \\
\hline Puy-de-Dôme ${ }^{(1)} \ldots \ldots \ldots \ldots$. . & $\begin{array}{l}\text { Sapin } \\
\text { Epicéa }\end{array}$ & $\begin{array}{l}208 \\
226\end{array}$ & - & $\begin{array}{l}1400 \\
1880\end{array}$ & $\begin{array}{l}57 \% \\
50 \%\end{array}$ \\
\hline
\end{tabular}

(1) Départements touchès par la tempête de novembre 1982.

En particulier. dans le Puy-de-Dôme, les chablis ont ète en gènéral commercialısès à l'unité de produrt avant l'automne. 


\section{LE PIN SYLVESTRE}

La mobilisation a atteint son niveau-record, en relation avec les effets de la tempête de novembre 1982 et l'importance des bois scolytés dans le Loiret.
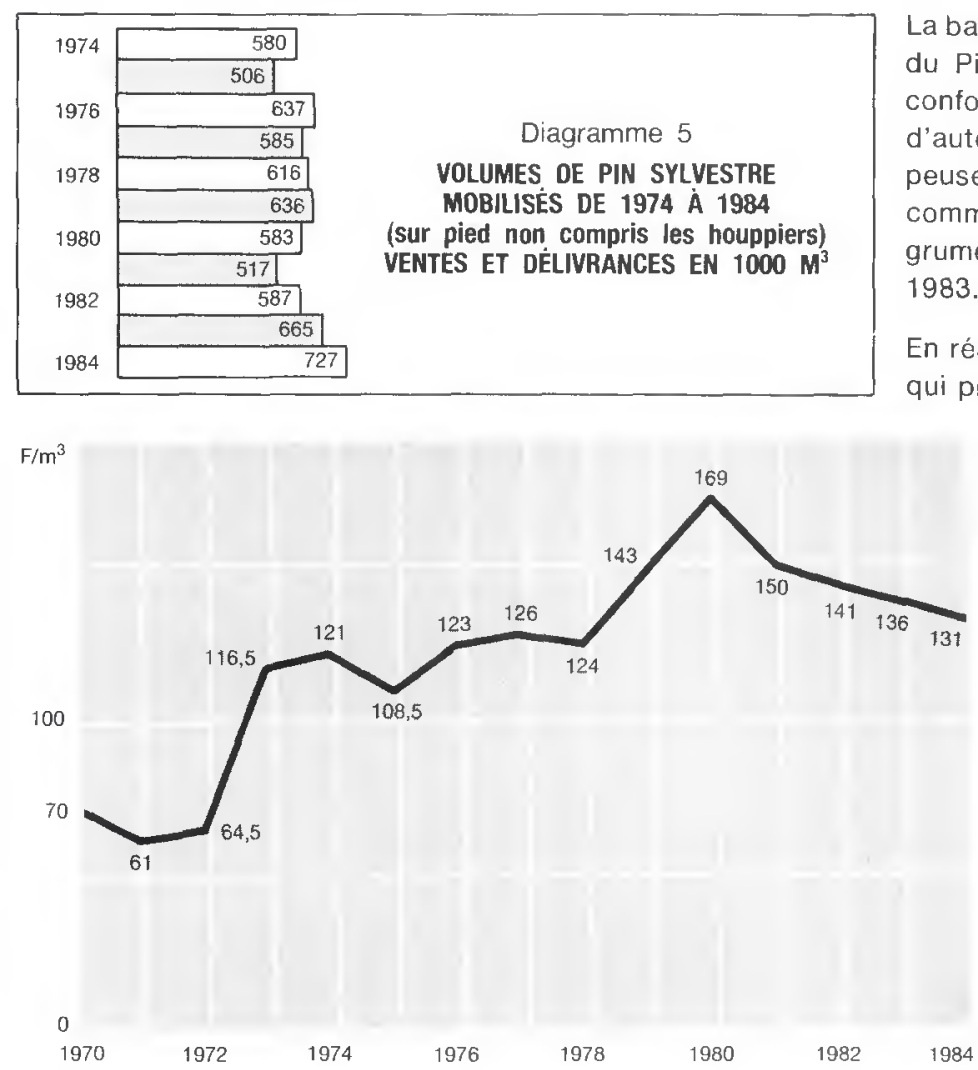

La baisse de $3,5 \%$ du prix moyen du Pin sylvestre tous diamètres confondus, obtenue aux ventes d'automne, est un peu trompeuse, dans la mesure où il a été commercialisé davantage de grumes de petits diamètres qu'en 1983.

En réalitè, c'est plutôt la stabilité qui prédomine dans les départements de plaine gros producteurs (cf. tableau VII). En montagne, la tendance a èté la même qu'en matière de Sapin $(-5$ à $10 \%$ ).

Graphique 6

PIN SYLVESTRE ĖVOLUTION DES PRIX MOYENS AUX GRANDES VENTES (taxe forfaitaire comprise)

Pin sylvestre de $25 \mathrm{~cm}$ et plus de diamètre Principaux départements producteurs - Grandes ventes 1984

\begin{tabular}{|c|c|c|c|c|}
\hline Départements & $\begin{array}{c}\text { Prix moyen } \\
1984\end{array}$ & $\begin{array}{c}\text { Variation } \\
1984-1983\end{array}$ & $\begin{array}{l}\text { Volume vendu } \\
\qquad\left(\mathrm{m}^{3}\right)\end{array}$ & $\begin{array}{l}\text { Proportion } \\
\text { d'invendus }\end{array}$ \\
\hline Loiret . . . . . . . . . . . . . & 169 & $+2 \%$ & 47790 & $6 \%$ \\
\hline Seine-et-Marne ..... & 100 & $-17 \%$ & 6920 & $59 \%$ \\
\hline Sarthe & 183 & $-6 \%$ & 8730 & $18 \%$ \\
\hline Orne .... & 263 & $+2 \%$ & 15320 & $11 \%$ \\
\hline Seine-Maritime/Eure . & 189 & $-4 \%$ & 26290 & $23 \%$ \\
\hline Puy-de-Dôme ${ }^{(1)}$ & 125 & pas de vente & 2840 & $39 \%$ \\
\hline Haute-Loire ${ }^{(1)}$ & 174 & en 1983 & 480 & $66 \%$ \\
\hline Vosges ................ & 147 & $-9 \%$ & 21505 & $35 \%$ \\
\hline
\end{tabular}

(1) Départements touchés par la tempête de novembre 1982: les chablis ont été commercialisés avant l'automne. 


\section{LE PIN MARITIME}

II s'agit d'un marchè étroitement localisè dans le Sud-Ouest et traditionnellement à part.
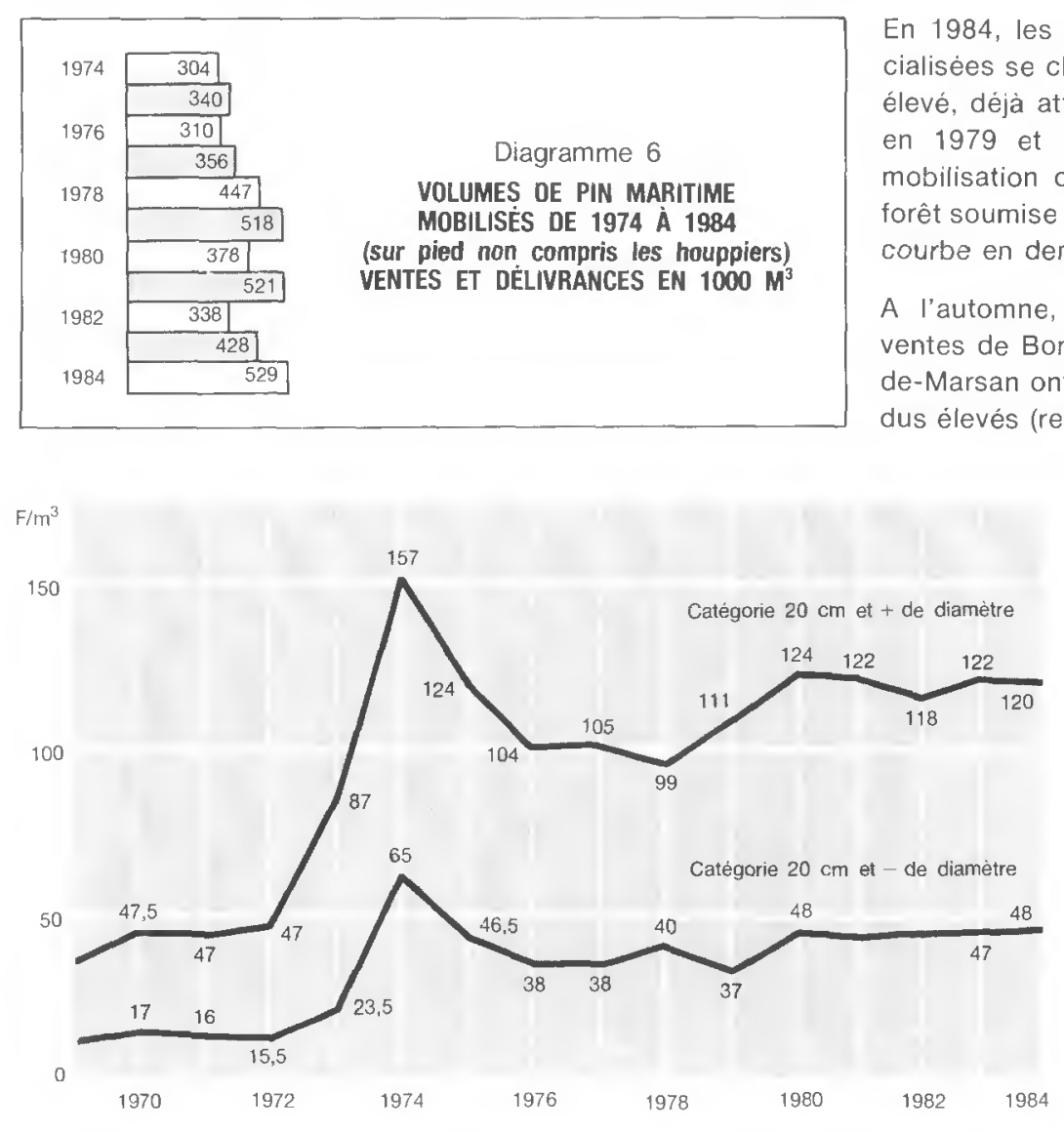

En 1984, les quantités commercialisèes se chiffrent à un niveau élevé, déjà atteint dans le passé en 1979 et 1981. En effet, la mobilisation du Pin maritime en forêt soumise a toujours suivi une courbe en dents de scie.

A l'automne, les rèsultats des ventes de Bordeaux et de Montde-Marsan ont traduit des invendus élevés (respectivement 28 et $48 \%$ ), mais plutôt moins élevés que d'habitude, et des prix stationnaires. A noter que la courbe des prix du Pin maritime stagne en francs courants depuis 1975.

Graphique 7

PIN MARITIME ÉVOLUTION OES PRIX MOYENS AUX GRANDES VENTES (taxe forfaitaire comprise)

\section{LES RÉSINEUX DIVERS}

Bien que traditionnellement difficile comme en témoigne le pourcentage des invendus à l'automne (cf. tableau VIII page 272), la commercialisation de ces rèsineux divers s'est situèe à un niveau plutôt èlevè en 1984 .

L'analyse des prix est toujours très délicate dans la mesure où les fluctuations de prix sont souvent davantage dues à des variations de conditions d'exploitation et de vidange ou de qualité des bois qu'à l'évolution de la conjoncture.

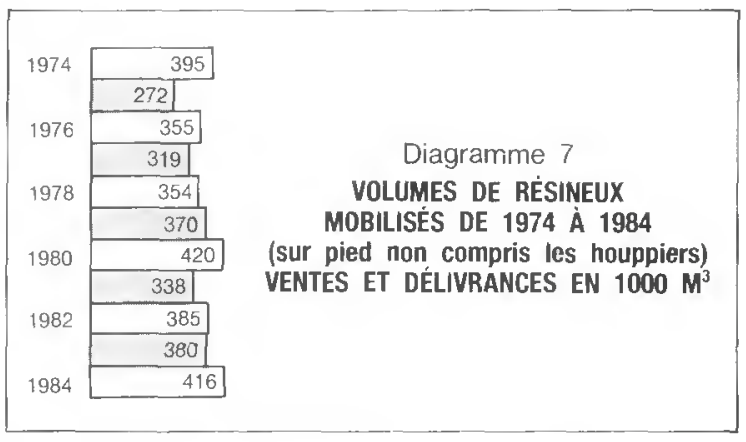




\begin{tabular}{|c|c|c|c|c|}
\hline Départements & $\begin{array}{c}\text { Prix moyen } \\
1984\end{array}$ & $\begin{array}{c}\text { Variation } \\
1984-1983\end{array}$ & $\begin{array}{c}\text { Volume vendu } \\
\qquad\left(\mathrm{m}^{3}\right)\end{array}$ & $\begin{array}{l}\text { Proportion } \\
\text { d'invendus }\end{array}$ \\
\hline Hautes-Alpes ${ }^{(1)} \ldots \ldots \ldots \ldots$. & 172 & $8 \%$ & 14790 & $58 \%$ \\
\hline Alpes-de-Haute-Provence ${ }^{(2)} \ldots$ & 100 & $+14 \%$ & 24500 & $34 \%$ \\
\hline Drôme ${ }^{(3)(*)} \ldots$ & 69 & $-9 \%$ & 2690 & $85 \%$ \\
\hline Lozère ${ }^{(4) \mid *)} \ldots \ldots .$. & 125 & $+9 \%$ & 23213 & $4 \%$ \\
\hline Pyrénèes-Orientales ${ }^{\{5\rangle}$. & 104 & $+28 \%$ & 5540 & $59 \%$ \\
\hline Corse $^{(6)}$ & 212 & $-29 \%$ & 11580 & $72 \%$ \\
\hline
\end{tabular}

(1) Essentıellement Melèze et Pin noir

(2) Essentiellement Pin noir, accessorrement Pin a crochets et Meleze

(3) Uniquement Pin noir.

14) Essentieliement PIn norr el Pin à crochets.

(5) Uniquement Pin a crochets.

(6) Uniquement Pin Iaricio.

(1) Departements touches par la tempête de novembre 1982.

\section{LES PETITS BOIS ET LES TAILLIS}

En matière de feuillus, l'écoulement s'est révélé plutôt facile à des prix fermes en liaison avec une demande de bois de trituration plutôt soutenue. Les volumes mobilisés en taillis sont en accroissement régulier depuis 1978 (sauf en 1983 où l'offre en était un peu moindre).

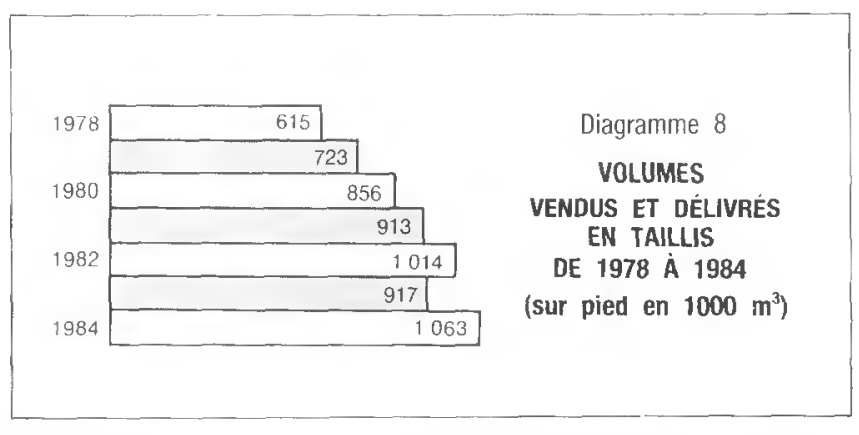

A noter que les délivrances directes aux communes propriétaires représentent $60 \%$ des quantités mobilisées en forêt communale pour. cette catégorie de produits.

\section{CONCLUSION}

Comme l'année 1983, l'année 1984 aura été marquée par un apport en volume particulièrement important sur le marché français du bois, lié en très grande partie aux conséquences des tempêtes répétées qui ont ravagé la France depuis novembre 1982. A tous ces chablis, constitués essentiellement de Sapin - Épicéa - Pin (Centre de la France) et de Hêtre et Chêne (moitié Nord de la France), il convient de rajouter en 1984 une mobilisation accrue en résineux dans le Loiret et en Franche-Comté (bois scolytés) ainsi qu'en Aquitaine (Pin maritime dont la mobilisation s'est toujours avérée irrégulière).

A l'exception du Chêne dont le marché est apparu très actif sous la pression de la demande étrangère, l'incidence de tous ces chablis n'a pas manqué de peser sur les prix des bois en 1984. 
II est vraisemblable que l'année 1985 sera encore perturbée par les conséquences de ces tempêtes puisque les chablis issus de la dernière de novembre 1984 sont mis sur le marché au début de cette année; de plus, les intempéries de janvier 1985 ont entraîné le gel de quantités de Pin maritime relativement importantes dans le Sud-Ouest.

OFFICE NATIONAL DES FORETS

Département commerciał

2. avenue de Saint-Mande

75570 PARIS CEDEX 12

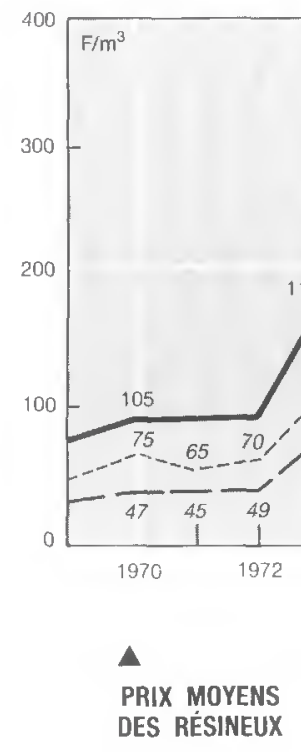

PRIX MOYENS

DES FEUILLUS

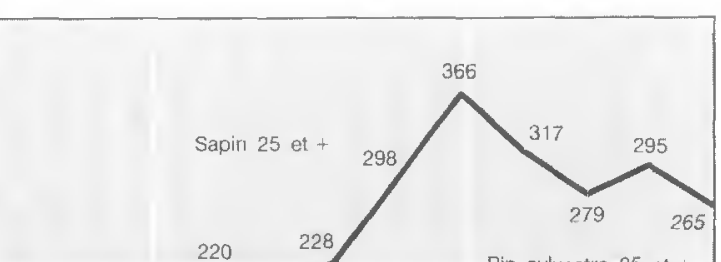

ANNEXE 1

GRANDES VENTES O.N.F.

Pin sylvestre 25 et +

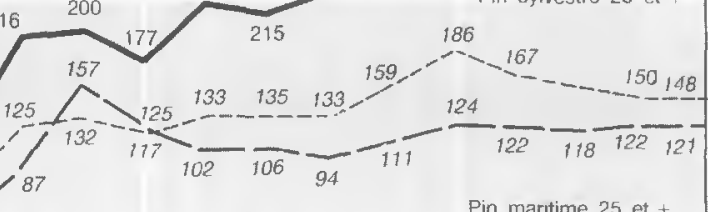

Pin maritime 25 et +
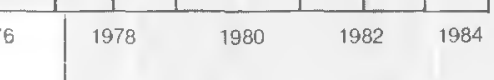

DES RÉSINEUX

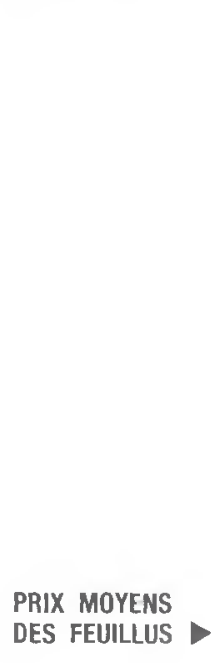

Picardie - Vosges) et de la reduction de l'offre d'automne dans ces régions. 
OFFICE NATIONAL DES FORÊTS

ANNEXE ॥

GRANDES VENTES 1984

\begin{tabular}{|c|c|c|c|c|c|c|}
\hline & $\begin{array}{c}\text { Volume } \\
\text { mis en } \\
\text { vente } \\
\left(1000 \mathrm{~m}^{3}\right)\end{array}$ & $\begin{array}{c}\begin{array}{c}\text { Volume } \\
\text { vendu }\end{array} \\
\left(1000 \mathrm{~m}^{3}\right)\end{array}$ & $\begin{array}{c}\begin{array}{c}\text { Volume } \\
\text { invendu }\end{array} \\
\left(1000 \mathrm{~m}^{3}\right)\end{array}$ & $\begin{array}{c}\text { Prix } \\
\text { moyen } \\
1984 \\
\mathrm{~F} / \mathrm{m}^{3}\end{array}$ & $\begin{array}{c}\text { Prix } \\
\text { moyen } \\
1983 \\
\mathrm{~F} / \mathrm{m}^{3}\end{array}$ & $\begin{array}{l}\text { Variation } \\
1984-1983\end{array}$ \\
\hline Chêne 50 et + & 502 & 451 & 51 & 804 & 687 & $+17 \%$ \\
\hline Chêne $30 / 45$ & 382 & 321 & 61 & 203 & 197 & $+3 \%$ \\
\hline Chêne 25 et - & 120 & 99 & 21 & 26 & 24 & +8 \\
\hline TOTAL CHÊNE & 1004 & 871 & 133 & 494 & 424 & $+17 \%$ \\
\hline Hêtre 40 et + & 675 & 524 & 151 & 231 & 277 & -17 \\
\hline Hêtre $30 / 35$ & 159 & 119 & 40 & 99,5 & 111 & -10 \\
\hline Hêtre 25 et - & 147 & 110 & 37 & 34 & 34 & - \\
\hline TOTAL HÊTRE & 981 & 753 & 228 & 181 & 219 & $-17 \%$ \\
\hline Peuplier & 28 & 21 & 7 & 165,5 & 155 & +7 \\
\hline Autres feuillus & 341 & 280 & 61 & 106,2 & 105 & $+1 \%$ \\
\hline TOTAL FEUILLUS & 2354 & 1925 & 429 & 312 & 284 & $+10 \%$ \\
\hline Sapin 25 et + & 873 & 639 & 234 & 265 & 295 & -10 \\
\hline Sapin 20 et - & 41 & 30 & 11 & 38,8 & 53 & -27 \\
\hline TOTAL SAPIN & 914 & 669 & 245 & 255 & 285 & $-11 \%$ \\
\hline Epicéa 25 et + & 455 & 319 & 136 & 264,7 & 289 & $-9 \%$ \\
\hline Epicéa 20 et - & 50 & 36 & 14 & 45,7 & 49 & -7 \\
\hline TOTAL ÉPICÉA & 505 & 355 & 150 & 242 & 269 & -10 \\
\hline Pin sylvestre 25 et + & 420 & 285 & 135 & 148 & 150 & -1 \\
\hline Pin sylvestre 20 et - & 73 & 43 & 30 & 20,8 & 18,8 & $+11 \%$ \\
\hline TOTAL PIN SYLVESTRE & 493 & 328 & 165 & 131 & 136 & -4 \\
\hline Pin maritime 25 et + & 265 & 153 & 112 & 120 & 122 & -2 \\
\hline Pin maritime 20 et - & 40 & 30 & 10 & 48 & 47 & +2 \\
\hline TOTAL PIN MARITIME & 305 & 183 & 122 & 108 & 112 & -3 \\
\hline Autres résineux 25 et + & 298 & 157 & 141 & 121 & 134 & -10 \\
\hline Autres résineux 20 et - & 98 & 48 & 50 & 21,4 & 20,5 & +4 \\
\hline TOTAL AUTRES RÉSINEUX & 396 & 205 & 191 & 97,7 & 105 & -7 \\
\hline TOTAL RÉSINEUX & 2613 & 1740 & 873 & 195 & 219 & $-11 \%$ \\
\hline Taillis feuillus & 299 & 229 & 70 & 32,1 & 28 & $+15 \%$ \\
\hline Houppiers feuillus & 894 & 727 & 167 & 12 & 12,1 & $-0,83 \%$ \\
\hline Houppiers résineux & 235 & 145 & 90 & 0,6 & 0,7 & -14 \\
\hline TOTAL GÉNÉRAL & 6395 & 4766 & 1629 & 200,5 & 198 & +1 \\
\hline
\end{tabular}


ANNEXE III

RÉSULTATS COMPARÉS DES GRANDES VENTES DEPUIS 10 ANS

\begin{tabular}{|c|c|c|c|c|c|c|}
\hline Années & Catégories (1) & $\begin{array}{l}\text { Volume } \\
\text { mis en } \\
\text { vente } \\
\left(1000 \mathrm{~m}^{3}\right)\end{array}$ & $\begin{array}{c}\text { Volume } \\
\text { vendu } \\
\left(1000 \mathrm{~m}^{3}\right)\end{array}$ & $\begin{array}{c}\text { Volume } \\
\text { invendu } \\
\left(1000 \mathrm{~m}^{3}\right)\end{array}$ & $\begin{array}{c}\% \\
\text { invendu }\end{array}$ & $\begin{array}{c}\text { Recettes } \\
\text { (millions } \\
\text { de F } \\
\text { courants) }\end{array}$ \\
\hline 1974 & $\begin{array}{l}\text { Feuillus } \\
\text { Résineux } \\
\text { TOTAL }\end{array}$ & $\begin{array}{l}3920 \\
2665 \\
6585\end{array}$ & $\begin{array}{l}3270 \\
2089 \\
5359\end{array}$ & $\begin{array}{r}650 \\
576 \\
1226\end{array}$ & $\begin{array}{l}16 \% \\
22 \% \\
19 \%\end{array}$ & 642,6 \\
\hline 1975 & $\begin{array}{l}\text { Feuillus } \\
\text { Résineux } \\
\text { TOTAL }\end{array}$ & $\begin{array}{l}3785 \\
2677 \\
6462\end{array}$ & $\begin{array}{l}2664 \\
1955 \\
4619\end{array}$ & $\begin{array}{r}1121 \\
722 \\
1843\end{array}$ & $\begin{array}{l}30 \% \\
27 \% \\
29 \%\end{array}$ & 500,5 \\
\hline 1976 & $\begin{array}{l}\text { Feuillus } \\
\text { Résineux } \\
\text { TOTAL }\end{array}$ & $\begin{array}{l}4191 \\
2982 \\
7173\end{array}$ & $\begin{array}{l}3653 \\
2445 \\
6098\end{array}$ & $\begin{array}{r}538 \\
537 \\
1075\end{array}$ & $\begin{array}{l}13 \% \\
18 \% \\
15 \%\end{array}$ & 791,1 \\
\hline 1977 & $\begin{array}{l}\text { Feuillus } \\
\text { Résineux } \\
\text { TOTAL }\end{array}$ & $\begin{array}{l}3677 \\
2690 \\
6367\end{array}$ & $\begin{array}{l}3318 \\
2179 \\
5497\end{array}$ & $\begin{array}{l}359 \\
511 \\
870\end{array}$ & $\begin{array}{l}10 \% \\
19 \% \\
14 \%\end{array}$ & 835,7 \\
\hline 1978 & $\begin{array}{l}\text { Feuillus } \\
\text { Résineux } \\
\text { TOTAL }\end{array}$ & $\begin{array}{l}3487 \\
2587 \\
6074\end{array}$ & $\begin{array}{l}3174 \\
2082 \\
5256\end{array}$ & $\begin{array}{l}313 \\
505 \\
818\end{array}$ & $\begin{array}{r}9 \% \\
20 \% \\
13 \%\end{array}$ & 915,3 \\
\hline 1979 & $\begin{array}{l}\text { Feuillus } \\
\text { Résineux } \\
\text { TOTAL }\end{array}$ & $\begin{array}{l}3512 \\
2744 \\
6256\end{array}$ & $\begin{array}{l}3261 \\
2369 \\
5630\end{array}$ & $\begin{array}{l}251 \\
375 \\
626\end{array}$ & $\begin{array}{r}7 \% \\
14 \% \\
10 \%\end{array}$ & 1121,3 \\
\hline 1980 & $\begin{array}{l}\text { Feuillus } \\
\text { Résineux } \\
\text { TOTAL }\end{array}$ & $\begin{array}{l}3297 \\
2661 \\
5958\end{array}$ & $\begin{array}{l}2754 \\
2250 \\
5004\end{array}$ & $\begin{array}{l}543 \\
411 \\
954\end{array}$ & $\begin{array}{l}16 \% \\
15 \% \\
16 \%\end{array}$ & 1099,8 \\
\hline 1981 & $\begin{array}{l}\text { Feuillus } \\
\text { Résineux } \\
\text { TOTAL }\end{array}$ & $\begin{array}{l}3684 \\
2768 \\
6452\end{array}$ & $\begin{array}{l}2791 \\
2015 \\
4806\end{array}$ & $\begin{array}{r}893 \\
753 \\
1646\end{array}$ & $\begin{array}{l}24 \% \\
27 \% \\
25 \%\end{array}$ & 914,6 \\
\hline 1982 & $\begin{array}{l}\text { Feuillus } \\
\text { Résineux } \\
\text { TOTAL }\end{array}$ & $\begin{array}{l}3821 \\
3009 \\
6830\end{array}$ & $\begin{array}{ll}3 & 018 \\
2 & 137 \\
5 & 155\end{array}$ & $\begin{array}{r}803 \\
872 \\
1675\end{array}$ & $\begin{array}{l}21 \% \\
29 \% \\
25 \%\end{array}$ & 937,4 \\
\hline 1983 & $\begin{array}{l}\text { Feuillus } \\
\text { Résineux } \\
\text { TOTAL }\end{array}$ & $\begin{array}{l}3746 \\
2440 \\
6186\end{array}$ & $\begin{array}{l}3049 \\
1770 \\
4819\end{array}$ & $\begin{array}{r}697 \\
670 \\
1367\end{array}$ & $\begin{array}{l}19 \% \\
27 \% \\
22 \%\end{array}$ & 954,9 \\
\hline 1984 & $\begin{array}{l}\text { Feuillus } \\
\text { Résineux } \\
\text { TOTAL }\end{array}$ & $\begin{array}{l}3546 \\
2848 \\
6394\end{array}$ & $\begin{array}{l}2881 \\
1885 \\
4766\end{array}$ & $\begin{array}{r}665 \\
963 \\
1628\end{array}$ & $\begin{array}{l}19 \% \\
34 \% \\
25 \%\end{array}$ & 963,2 \\
\hline
\end{tabular}

(1) Les volumes de feuillus et resineux s'entendent toutes catègories comprises. 
OFFICE NATIONAL DES FORÊTS

ANNEXE IV L'ÉVOLUTION DE LA MOBILISATION DE BOIS EN PROVENANCE DE LA FORÊT SOUMISE

\begin{tabular}{|c|c|c|c|c|c|c|}
\hline & \multicolumn{3}{|c|}{ Offre totale bois sur pied } & \multirow{2}{*}{$\begin{array}{l}\text { Volume } \\
\text { mobilisé } \\
\text { bois } \\
\text { sur pied }\end{array}$} & \multirow{2}{*}{$\begin{array}{l}\text { Invendus } \\
\text { au } 31 / 12 \\
\text { année n }\end{array}$} & \multirow{2}{*}{$\begin{array}{c}\text { Volume } \\
\text { mobilisé } \\
\text { bois } \\
\text { façonnés }\end{array}$} \\
\hline & $\begin{array}{c}\text { Invendus } \\
\text { au } 31 / 12 \\
\text { année } n-1\end{array}$ & Martelages & Total & & & \\
\hline 1974 & 350 & 8356 & 8706 & 7736 & 970 & 1530 \\
\hline 1975 & 970 & 8323 & 9293 & 7623 & 1670 & 1599 \\
\hline 1976 & 1670 & 8453 & 10123 & 9183 & 940 & 1581 \\
\hline 1977 & 940 & 8564 & 9504 & 8764 & 740 & 1704 \\
\hline 1978 & 740 & 8759 & 9499 & 8854 & 645 & 1677 \\
\hline 1979 & 645 & 8653 & 9298 & 8842 & 456 & 1842 \\
\hline 1980 & 456 & 8993 & 9449 & 8593 & 856 & 1883 \\
\hline 1981 & 856 & 9211 & 10067 & 8617 & 1450 & 1856 \\
\hline 1982 & 1450 & 9432 & 10882 & 9435 & 1447 & 1863 \\
\hline $1983^{(+1}$ & 1447 & 10465 & 11912 & 10747 & 1165 & 1925 \\
\hline $1984^{(*)}$ & 1165 & 11685 & 12850 & 11478 & 1372 & 1973 \\
\hline
\end{tabular}

(*) Annèes fortement perturbèes par les chabis Issus de la tempète de novembre 1982 et mis sur le marché en 1983 et par les chablis issus des tempëtes de février el jullet 1984.

LA MOBILISATION OES BOIS EN FORĖTS SOUMISES AU RÉgIME FORESTIER EN 1984 ET LES PRIX OBTENUS AUX GRANDES VENTES OE L'AUTOMNE 1984 (Rèsume)

Pour la troisième année consécutive, les quantites de bois mobilisees en provenance des toréts soumises au iégime torestier ont atteint un niveau exceptionnel - soit $13450000 \mathrm{~m}^{3}$, en raison de l'abondance des chabtis issus de tempetes successives iNord-Duest en revrier. Vosges en juillet et á nouveau Nord-0uest en novembrel et des bois depérissants

L'otfre globale de bols en 1984 avec un volume de $14800000 \mathrm{~m}^{3}$ aura finalement dépasse celle de 1983. pourtant deja partıculièrement elevée. Cependant. le stock d'invendus en fin d'annee - $1370000 \mathrm{~m}^{3}$ — tout en étant un peu plus important que celui de 1983 - $1165000 \mathrm{~m}^{3}$ - reste inférıeur á celur des deux annees 1982-1983-1450000 $\mathrm{m}^{3}$

Les recettes-bols (en trancs courants), tant domaniales que des collectivites publiques, ont legerement progresse par rapport á 1983. en rason de laugmentatron des volumes mobilises et de la hausse des prix du chëre. d'une part. et malgre la baisse des prix de nombreuses essences selon la conjoncture et la "decoto-chablis", d'autre part

THE 1984 TRAOE IN WOOO COMING FROM FORESTS UNDER STATE CONTROL, AND TME PRICES OBTAINEO AT THE MAIN AUTUMN SALES OF 1984 (Summary)

For the third year in succession, the quantities of wood put on the market from the forests under state control reached an exceptionally high figure $13450000 \mathrm{~m}^{3}$ — because of the large amounts of wind thrown timber Itrom the storms that succeeded one another, in the North-west in February. the Vosges in July, and again in the North-west in Novembery and from dying trees

The grand total otfered for sale in 1984, with a volume of $1.4800000 \mathrm{~m}^{3}$. ended up by exceeding that of 1983, although that was exceptionally high. However, the unsoid stock at the end of the year $\left(1370000 \mathrm{~m}^{3}\right.$ i while being a little greater than that of $1983\left(1,165000 \mathrm{~m}^{3}\right)$. Was still less than that of the two years $1982-1983\left(1450000 \mathrm{~m}^{3}\right)$.

The income from sales of wood, at actual monetary values, both in the slate forests and in those belonging to other public bodies, was slightly up compared with 1983 on account of the increase in the volume soid and the rise in oak prices - in spite of these being offset by lower prices for many other species as a result of the economic situation, and by the "windthrow discount

OIE HOLZAUFBRINGUNG IN OEN BEFÖRSTERTEN WÄLOERN IM JAHR 1985 UNO OIE BEI OEN GROSSEN HERBSTVERKÄUFEN 1984 ERZIELTEN PREISE (Zusammenfassung)

Zum dritten Mai innerhalb von drei Jahren haben die aus den beforsterten Wädern aufgebrachten Holzmengen ein aussergewahniliches Niveau erreicht, d.h. $13450000 \mathrm{~m}^{3}$ infalge der grossen Mengen an Windbruch nach den aufenanderfolgenden Sturmen (Nordwestrankreich am Februar, Vogesen im Juli und erneut der Nordwesten im November) und infolge der absterbenden Holzer.

Das Gesamtangebol an Holz lag 1984 mit $14800000 \mathrm{~m}^{3}$ schliesshch noch hoher ais im Jahre 1983. das bereits en besonders hohes Angebot aufwies Injessen liegt die am Jahresende unverkaufte Holzmenge mi $1370000 \mathrm{~m}^{3}$ - obwolh etwas grosser als $1983\left(1165000 \mathrm{~m}^{3}\right)$ - niedriger als die der Jahre $1982-1983\left(1450000 \mathrm{~m}^{3}\right)$

Die Holzeinnahmen, (in laufenden Francs), sowohl aus staatichen als auch aus kommunalen Waldern. Sind leicht angestiegen im vergleich zu 1983 infolge des grhohten Angebots und des Preisanstiegs fur Eiche und dies trotz der Pretssenkung zahlrercher Holzarten gemass der Marktlage und der herabgesetzten Preise fur Windbruch.

LA MOVILIZACION DE LAS MADERAS EN LOS BOSQUES SOMETIOOS AL REGIMEN FORESTAL EN 1984 Y LOS PRECIOS OBTENIOOS EN LAS GRANOES VENTAS OEL OTONO OE 1984 (Resumeri)

Por lercer ano consecutivo. las cantidades de madera movilizadas. procedentes de bosques sometıdos al regimen forestal, han logrado un nivel excepcional es decir $13450000 \mathrm{~m}^{3}$ - en razón de la abunancia de los arboles lumbados por las tempestades sucesivas iNoideste en Febrero, vosgos en Juilo y nuevamente Noroeste en Noviembrei y maderas deteriørados

La oterta global de madera en 1984 , con un volumen de $14800000 \mathrm{~m}^{3}$ habra sobrepasado finalmente la de 1983, va de por si particularmente elevada Sir embarçu la resevra de la no vendida al fin del ano - $1370000 \mathrm{~m}^{3}$ - aun siendo un poco mas importante que la de 1983 - $1175000 \mathrm{~m}^{3}$ - es inferıor a la de los dos anos $1982 / 1983-1450000 \mathrm{~m}^{3}$

Los ingresos-madera ien francos actuales), tanto conunales como de las colectividades publicas, han progresado ligeramente con relacion a 1983 , a causa del aumento de los volumenes movilizados y de la alza de los piecios del Roble de una parte y a pesar de la baja de los precios de numerosas varıddes. segun la coyuntura y la depreciacion por derribo, de otra parte. 


\section{CALENDRIER \\ DES GRANDES VENTES D'AUTOMNE 1985}

\begin{tabular}{|c|c|c|c|}
\hline DATES & HEURES & LIEUX DE VENTE & RÉGIONS \\
\hline 16 Septembre & $8 \mathrm{~h}$ & Màcon & BOURGOGNE \\
\hline $\begin{array}{l}17 \text { Septembre } \\
17 \text { Septembre }\end{array}$ & $\begin{array}{l}8 \mathrm{~h} \\
8 \mathrm{~h} 30\end{array}$ & $\begin{array}{l}\text { Pontarlier } \\
\text { Digne }\end{array}$ & $\begin{array}{l}\text { FRANCHE-COMTÉ } \\
\text { PROVENCE-ALPES-CÔTE D'AZUR }\end{array}$ \\
\hline 18 Septembre & $10 \mathrm{~h}$ & Briey & LORRAINE \\
\hline $\begin{array}{l}19 \text { Septembre } \\
19 \text { Septembre } \\
19 \text { Septembre } \\
19 \text { Septembre }\end{array}$ & $\begin{array}{l}8 \mathrm{~h} \\
8 \mathrm{~h} 30 \\
9 \mathrm{~h} \\
9 \mathrm{~h}\end{array}$ & $\begin{array}{l}\text { Dôle } \\
\text { Gap } \\
\text { Laon } \\
\text { Thonon }\end{array}$ & $\begin{array}{l}\text { FRANCHE-COMTÉ } \\
\text { PROVENCE-ALPES-CÔTE D'AZUR } \\
\text { PICARDIE } \\
\text { RHÔNE-ALPES }\end{array}$ \\
\hline $\begin{array}{l}20 \text { Septembre } \\
20 \text { Septembre } \\
20 \text { Septembre }\end{array}$ & $\begin{array}{l}8 \mathrm{~h} \\
8 \mathrm{~h} \\
9 \mathrm{~h} 30\end{array}$ & $\begin{array}{l}\text { Beaune } \\
\text { Epinal-Sud } \\
\text { Belfort }\end{array}$ & $\begin{array}{l}\text { BOURGOGNE } \\
\text { LORRAINE } \\
\text { FRANCHE-COMTÉ }\end{array}$ \\
\hline 23 Septembre & $8 \mathrm{~h}$ & Besançon & FRANCHE-COMTÉ \\
\hline $\begin{array}{l}24 \text { Septembre } \\
24 \text { Septembre } \\
24 \text { Septembre } \\
24 \text { Septembre } \\
24 \text { Septembre }\end{array}$ & $\begin{array}{l}8 \mathrm{~h} \\
8 \mathrm{~h} 30 \\
9 \mathrm{~h} 30 \\
10 \mathrm{~h} \\
15 \mathrm{~h}\end{array}$ & $\begin{array}{l}\text { Dijon-Est } \\
\text { Raon-l'Etape } \\
\text { Aubenas } \\
\text { Nice } \\
\text { Dax }\end{array}$ & $\begin{array}{l}\text { BOURGOGNE } \\
\text { LORRAINE } \\
\text { RHÔNE-ALPES } \\
\text { PROVENCE-ALPES-CÔTE D'AZUR } \\
\text { AQUITAINE }\end{array}$ \\
\hline $\begin{array}{l}25 \text { Septembre } \\
25 \text { Septembre } \\
25 \text { Septembre } \\
25 \text { Septembre }\end{array}$ & $\begin{array}{l}8 h \\
8 h 30 \\
9 h \\
10 h\end{array}$ & $\begin{array}{l}\text { Saint-Claude } \\
\text { Langres } \\
\text { Ajaccio } \\
\text { Guéret }\end{array}$ & $\begin{array}{l}\text { FRANCHE-COMTÉ } \\
\text { CHAMPAGNE-ARDENNE } \\
\text { CORSE } \\
\text { LIMOUSIN }\end{array}$ \\
\hline $\begin{array}{l}26 \text { Septembre } \\
26 \text { Septembre } \\
26 \text { Septembre }\end{array}$ & $\begin{array}{l}8 \text { Һ } 30 \\
9 \text { h } \\
9 \text { Һ } 30\end{array}$ & $\begin{array}{l}\text { Grenoble Est-Ouest } \\
\text { Lure } \\
\text { Avignon }\end{array}$ & $\begin{array}{l}\text { RHÔNE-ALPES } \\
\text { FRANCHE-COMTÉ } \\
\text { PROVENCE-ALPES-CÔTE D'AZUR }\end{array}$ \\
\hline $\begin{array}{l}27 \text { Septembre } \\
27 \text { Septembre } \\
27 \text { Septembre }\end{array}$ & $\begin{array}{l}8 \mathrm{~h} \\
8 \mathrm{~h} \\
9 \mathrm{~h}\end{array}$ & $\begin{array}{l}\text { Nantua } \\
\text { Neufchâteau } \\
\text { Blois }\end{array}$ & $\begin{array}{l}\text { RHÔNE-ALPES } \\
\text { LORRAINE } \\
\text { CENTRE }\end{array}$ \\
\hline $\begin{array}{l}30 \text { Septembre } \\
30 \text { Septembre } \\
30 \text { Septembre }\end{array}$ & $\begin{aligned} 8 \mathrm{~h} \\
9 \mathrm{~h} \\
10 \mathrm{~h}\end{aligned}$ & $\begin{array}{l}\text { Châtillon-sur-Seine } \\
\text { Villers-Cotterêts } \\
\text { Nantes }\end{array}$ & $\begin{array}{l}\text { BOURGOGNE } \\
\text { PICARDIE } \\
\text { PAYS-DE-LA-LOIRE }\end{array}$ \\
\hline $\begin{array}{l}1^{\text {er }} \text { Octobre } \\
1^{\text {er }} \text { Octobre } \\
1^{\text {er }} \text { Octobre } \\
1^{\text {er Octobre }}\end{array}$ & $\begin{array}{l}8 \mathrm{~h} \\
8 \mathrm{~h} 30 \\
9 \mathrm{~h} \\
10 \mathrm{~h}\end{array}$ & $\begin{array}{l}\text { Montbéliard } \\
\text { Charleville } \\
\text { Grenoble-Nord } \\
\text { Toulon }\end{array}$ & $\begin{array}{l}\text { FRANCHE-COMTÉ } \\
\text { CHAMPAGNE-ARDENNE } \\
\text { RHOONE-ALPES } \\
\text { PROVENCE-ALPES-CÔTE D'AZUR }\end{array}$ \\
\hline $\begin{array}{ll}2 & \text { Octobre } \\
2 & \text { Octobre } \\
2 & \text { Octobre } \\
2 & \text { Octobre } \\
2 & \text { Octobre } \\
2 & \text { Octobre }\end{array}$ & $\begin{array}{l}8 \mathrm{~h} \\
8 \mathrm{~h} \\
9 \mathrm{~h} \\
9 \mathrm{~h} \\
10 \mathrm{~h} \\
10 \mathrm{~h} 30\end{array}$ & $\begin{array}{l}\text { Gray } \\
\text { Remiremont } \\
\text { Fontainebleau } \\
\text { Mende } \\
\text { Angers } \\
\text { Bayeux }\end{array}$ & $\begin{array}{l}\text { FRANCHE-COMTÉ } \\
\text { LORRAINE } \\
\text { ILE-DE-FRANCE } \\
\text { LANGUEDOC-ROUSSILLON } \\
\text { PAYS-DE-LA-LOIRE } \\
\text { NORMANDIE }\end{array}$ \\
\hline $\begin{array}{ll}3 & \text { Octobre } \\
3 & \text { Octobre } \\
3 & \text { Octobre } \\
3 & \text { Octobre }\end{array}$ & $\begin{array}{l}8 \mathrm{~h} 30 \\
9 \mathrm{~h} \\
9 \mathrm{~h} 30 \\
14 \mathrm{~h} 30\end{array}$ & $\begin{array}{l}\text { Châlons-sur-Marne } \\
\text { Albertville } \\
\text { Tours } \\
\text { Le Puy }\end{array}$ & $\begin{array}{l}\text { CHAMPAGNE-ARDENNE } \\
\text { RHONEE-ALPES } \\
\text { CENTRE } \\
\text { AUVERGNE }\end{array}$ \\
\hline $\begin{array}{ll}4 & \text { Octobre } \\
4 & \text { Octobre } \\
4 & \text { Octobre } \\
4 & \text { Octobre } \\
4 & \text { Octobre } \\
4 & \text { Octobre }\end{array}$ & $\begin{array}{l}8 \mathrm{~h} \\
8 \mathrm{~h} \\
8 \mathrm{~h} 30 \\
9 \mathrm{~h} \\
9 \mathrm{~h} 30 \\
10 \mathrm{~h}\end{array}$ & $\begin{array}{l}\text { Lunéville } \\
\text { Nevers } \\
\text { Valence } \\
\text { Valenciennes } \\
\text { Lons-le-Saunier } \\
\text { La Roche-sur-Yon }\end{array}$ & $\begin{array}{l}\text { LORRAINE } \\
\text { BOURGOGNE } \\
\text { RHÔNE-ALPES } \\
\text { NORD-PAS-DE-CALAIS } \\
\text { FRANCHE-COMTÉ } \\
\text { PAYS-DE-LA-LOIRE }\end{array}$ \\
\hline
\end{tabular}




\section{LIEUX, DATES ET HEURES D}

\begin{tabular}{|lrr|}
\hline \multicolumn{3}{|c|}{ NORMANDIE } \\
Bayeux & $02-10$, & $10 \mathrm{~h} 30$ \\
Rouen & $09-10$, & $9 \mathrm{~h} 00$ \\
Alençon & $14-10$, & $8 \mathrm{~h} 30$ \\
Dieppe & $23-10$, & $9 \mathrm{~h} 00$ \\
Lyons-la-Forêt & $30-10$, & $9 \mathrm{~h} 30$ \\
\hline
\end{tabular}

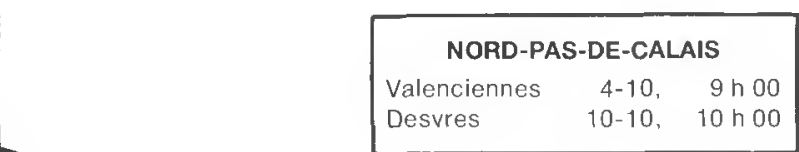

ILE-DE-FRANCE

Fontainebleau 02-10,

Versailles

14 h 00

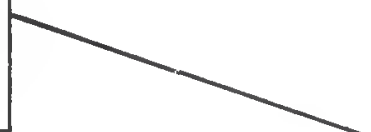

\begin{tabular}{|lrr|}
\hline \multicolumn{3}{c}{ CENTRE } \\
Blois & $27-09$, & $9 \mathrm{~h} 00$ \\
Tours & $03-10$, & $9 \mathrm{~h} 30$ \\
Châteauroux & $08-10$, & $9 \mathrm{~h} 30$ \\
Chartres & $11-10$, & $10 \mathrm{~h} 00$ \\
Orléans & $17-10$, & $8 \mathrm{~h} 00$ \\
Bourges & $21-10$, & $9 \mathrm{~h} 00$ \\
\hline
\end{tabular}

PAYS-DE-LA-LOIRE - BRETAGNE

Nantes

Angers

30-09,

02-10,

$10 \mathrm{~h} 00$

La Roche/Yon

Rennes

$04-10$

$10 \mathrm{~h} 00$

Le Mans

09-10,

10-10,

$10 \mathrm{~h} 00$

$8 \mathrm{~h} 30$

8 h 30

年

\section{POITOU-CHARENTES-LIMOUSIN}

Guéret

Poitiers

La Rochelle

25-09, $\quad 10$ h 00

Niort

$09-10$, 9 h 00

$11-10, \quad 10 \mathrm{~h} 30$

$14-10, \quad 10$ h 30

\begin{tabular}{|lrr|}
\hline \multicolumn{3}{|c|}{ AUVERGNE } \\
Le Puy & $03-10$, & 14 h 30 \\
Clermont-Ferrand $08-10$, & 9 h 00 \\
Aurillac & $10-10$, & 9 h 30 \\
Cerilly & $15-10$, & 9 h 00 \\
\hline
\end{tabular}

\begin{tabular}{|lrr|}
\hline \multicolumn{4}{|c|}{ AQUitAINE } \\
Dax & $24-09$, & 15 h 00 \\
Bayonne & $10-10$, & 15 h 00 \\
Pau & $16-10$, & 9 h 00 \\
Mont-de-Marsan30-10, & 14 h 15 \\
\hline
\end{tabular}

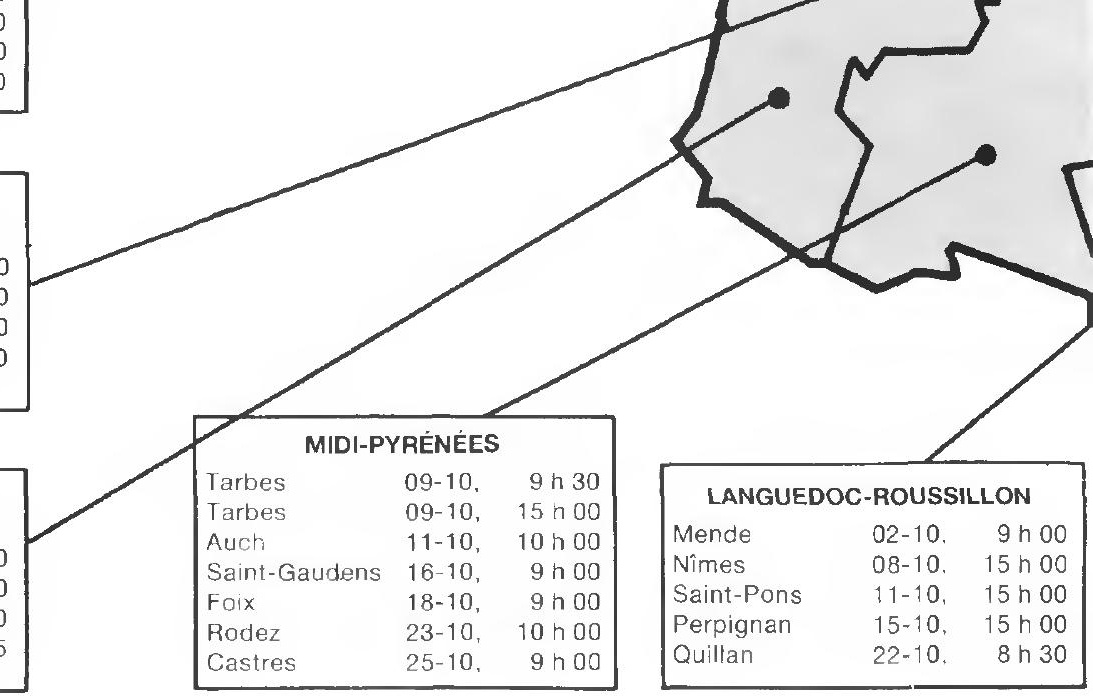




\section{/ENTE PAR RÉGIONS}

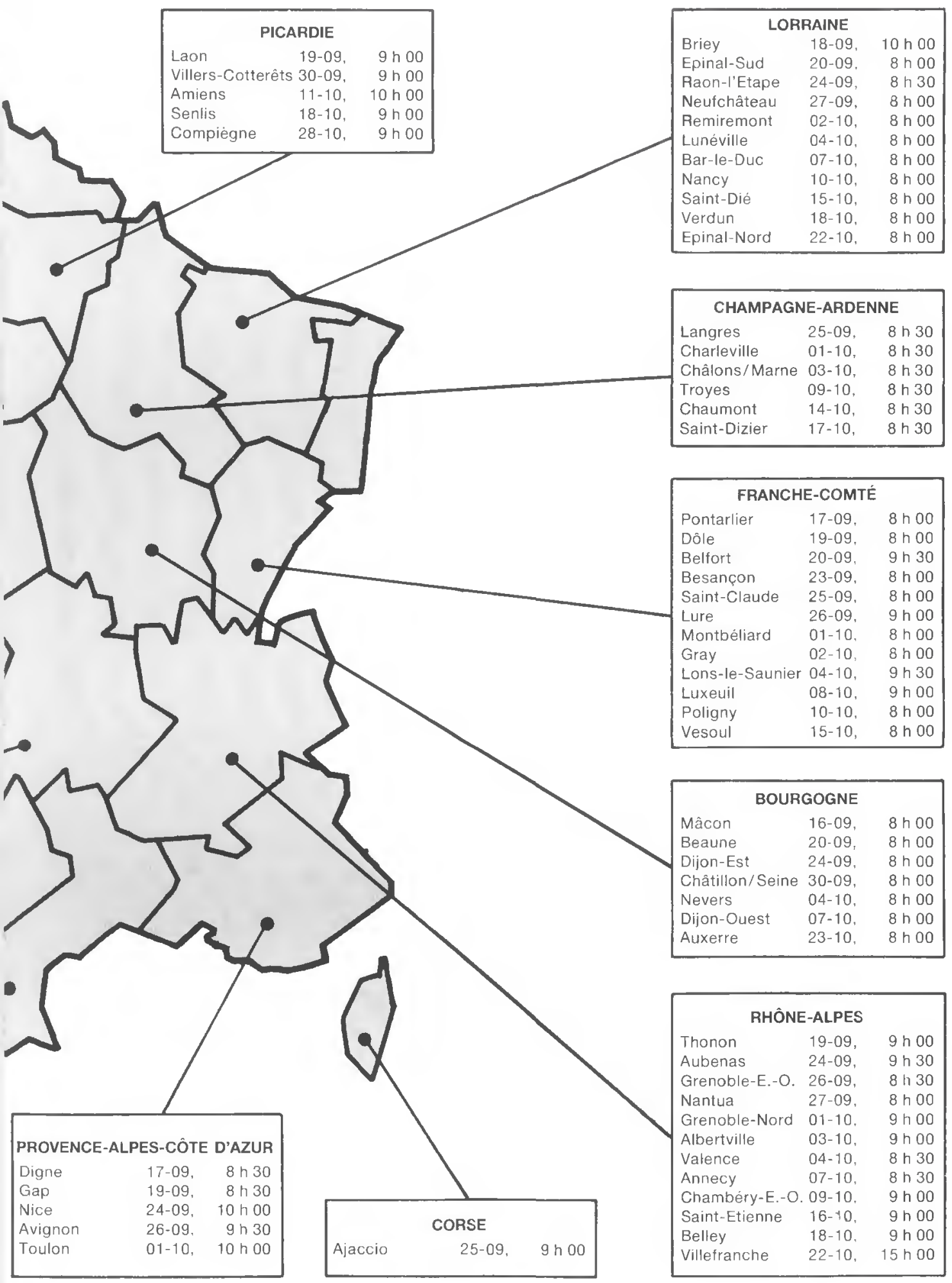


CALENDRIER DES GRANDES VENTES D'AUTOMNE 1985

\begin{tabular}{|c|c|c|c|}
\hline DATES & HEURES & LIEUX DE VENTE & RÉGIONS \\
\hline $\begin{array}{ll}7 & \text { Octobre } \\
7 & \text { Octobre } \\
7 & \text { Octobre }\end{array}$ & $\begin{array}{l}8 \mathrm{~h} \\
8 \mathrm{~h} \\
8 \mathrm{~h} 30\end{array}$ & $\begin{array}{l}\text { Bar-le-Duc } \\
\text { Dijon-Ouest } \\
\text { Annecy }\end{array}$ & $\begin{array}{l}\text { LORRAINE } \\
\text { BOURGOGNE } \\
\text { RHÔNE-ALPES }\end{array}$ \\
\hline $\begin{array}{ll}8 & \text { Octobre } \\
8 & \text { Octobre } \\
8 & \text { Octobre } \\
8 & \text { Octobre }\end{array}$ & $\begin{array}{l}9 \mathrm{~h} \\
9 \mathrm{~h} \\
9 \mathrm{~h} 30 \\
15 \mathrm{~h}\end{array}$ & $\begin{array}{l}\text { Clermont-Ferrand } \\
\text { Luxeuil } \\
\text { Châteauroux } \\
\text { Nimes }\end{array}$ & $\begin{array}{l}\text { AUVERGNE } \\
\text { FRANCHE-COMTÉ } \\
\text { CENTRE } \\
\text { LANGUEDOC-ROUSSILLON }\end{array}$ \\
\hline $\begin{array}{l}9 \text { Octobre } \\
9 \text { Octobre } \\
9 \text { Octobre } \\
9 \text { Octobre } \\
9 \text { Octobre } \\
9 \text { Octobre } \\
9 \text { Octobre }\end{array}$ & $\begin{array}{l}8 \mathrm{~h} 30 \\
8 \mathrm{~h} 30 \\
9 \mathrm{~h} \\
9 \mathrm{~h} \\
9 \mathrm{~h} \\
9 \mathrm{~h} 30 \\
15 \mathrm{~h}\end{array}$ & $\begin{array}{l}\text { Troyes } \\
\text { Rennes } \\
\text { Chambejry Est-Ouest } \\
\text { Poitiers } \\
\text { Rouen } \\
\text { Tarbes } \\
\text { Tarbes }\end{array}$ & $\begin{array}{l}\text { CHAMPAGNE-ARDENNE } \\
\text { BRETAGNE } \\
\text { RHÔNE-ALPES } \\
\text { POITOU-CHARENTES } \\
\text { NORMANDIE } \\
\text { MIDI-PYRÉNÉES } \\
\text { MIDI-PYRĖNEEES }\end{array}$ \\
\hline $\begin{array}{l}10 \text { Octobre } \\
10 \text { Octobre } \\
10 \text { Octobre } \\
10 \text { Octobre } \\
10 \text { Octobre } \\
10 \text { Octobre }\end{array}$ & $\begin{array}{l}8 \mathrm{~h} \\
8 \mathrm{~h} \\
8 \mathrm{~h} 30 \\
9 \mathrm{~h} 30 \\
10 \mathrm{~h} \\
15 \mathrm{~h}\end{array}$ & $\begin{array}{l}\text { Nancy } \\
\text { Poligny } \\
\text { Le Mans } \\
\text { Aurillac } \\
\text { Desvres } \\
\text { Bayonne }\end{array}$ & $\begin{array}{l}\text { LORRAINE } \\
\text { FRANCHE-COMTÉ } \\
\text { PAYS-DE-LA-LOIRE } \\
\text { AUVERGNE } \\
\text { NORD-PAS-DE-CALAIS } \\
\text { AQUITAINE }\end{array}$ \\
\hline $\begin{array}{l}11 \text { Octobre } \\
11 \text { Octobre } \\
11 \text { Octobre } \\
11 \text { Octobre } \\
11 \text { Octobre }\end{array}$ & $\begin{array}{l}10 \mathrm{~h} \\
10 \mathrm{~h} \\
10 \mathrm{~h} \\
10 \mathrm{~h} 30 \\
15 \mathrm{~h}\end{array}$ & $\begin{array}{l}\text { Amiens } \\
\text { Auch } \\
\text { Chartres } \\
\text { La Rochelle } \\
\text { Saint-Pons }\end{array}$ & $\begin{array}{l}\text { PICARDIE } \\
\text { MIDI-PYRÉNÉES } \\
\text { CENTRE } \\
\text { POITOU-CHARENTES } \\
\text { LANGUEDOC-ROUSSILLON }\end{array}$ \\
\hline $\begin{array}{l}14 \text { Octobre } \\
14 \text { Octobre } \\
14 \text { Octobre }\end{array}$ & $\begin{array}{r}8 \text { h } 30 \\
8 \text { h } 30 \\
10 \text { h } 30\end{array}$ & $\begin{array}{l}\text { Alençon } \\
\text { Chaumont } \\
\text { Niort }\end{array}$ & $\begin{array}{l}\text { NORMANDIE } \\
\text { CHAMPAGNE-ARDENNE } \\
\text { POITOU-CHARENTES }\end{array}$ \\
\hline $\begin{array}{l}15 \text { Octobre } \\
15 \text { Octobre } \\
15 \text { Octobre } \\
15 \text { Octobre }\end{array}$ & $\begin{array}{r}8 \mathrm{~h} \\
8 \mathrm{~h} \\
9 \mathrm{~h} \\
15 \mathrm{~h}\end{array}$ & $\begin{array}{l}\text { Saint-Dié } \\
\text { Vesoul } \\
\text { Cerilly } \\
\text { Perpignan }\end{array}$ & $\begin{array}{l}\text { LORRAINE } \\
\text { FRANCHE-COMTE } \\
\text { AUVERGNE } \\
\text { LANGUEDOC-ROUSSILLON }\end{array}$ \\
\hline $\begin{array}{l}16 \text { Octobre } \\
16 \text { Octobre } \\
16 \text { Octobre } \\
16 \text { Octobre }\end{array}$ & $\begin{array}{r}9 \mathrm{~h} \\
9 \mathrm{~h} \\
9 \mathrm{~h} \\
14 \mathrm{~h}\end{array}$ & $\begin{array}{l}\text { Pau } \\
\text { Saint-Etienne } \\
\text { Saint-Gaudens } \\
\text { Versailles }\end{array}$ & $\begin{array}{l}\text { AQUITAINE } \\
\text { RHÔNE-ALPES } \\
\text { MIDI-PYRÉNÉES } \\
\text { ILE-DE-FRANCE }\end{array}$ \\
\hline $\begin{array}{l}17 \text { Octobre } \\
17 \text { Octobre }\end{array}$ & $\begin{array}{l}8 \mathrm{~h} \\
8 \mathrm{~h} 30\end{array}$ & $\begin{array}{l}\text { Orlèans } \\
\text { Saint-Dizier }\end{array}$ & $\begin{array}{l}\text { CENTRE } \\
\text { CHAMPAGNE-ARDENNE }\end{array}$ \\
\hline $\begin{array}{l}18 \text { Octobre } \\
18 \text { Octobre } \\
18 \text { Octobre } \\
18 \text { Octobre }\end{array}$ & $\begin{array}{l}8 \mathrm{~h} \\
9 \mathrm{~h} \\
9 \mathrm{~h} \\
9 \mathrm{~h}\end{array}$ & $\begin{array}{l}\text { Verdun } \\
\text { Belley } \\
\text { Foix } \\
\text { Senlis }\end{array}$ & $\begin{array}{l}\text { LORRAINE } \\
\text { RHÔNE-ALPES } \\
\text { MIDI-PYRÉNÉES } \\
\text { PICARDIE }\end{array}$ \\
\hline 21 Octobre & $9 \mathrm{~h}$ & Bourges & CENTRE \\
\hline $\begin{array}{l}22 \text { Octobre } \\
22 \text { Octobre } \\
22 \text { Octobre }\end{array}$ & $\begin{array}{l}8 \mathrm{~h} \\
8 \mathrm{~h} 30 \\
15 \mathrm{~h}\end{array}$ & $\begin{array}{l}\text { Epinal-Nord } \\
\text { Quillan } \\
\text { Villefranche }\end{array}$ & $\begin{array}{l}\text { LORRAINE } \\
\text { LANGUEDOC-ROUSSILLON } \\
\text { RHONE-ALPES }\end{array}$ \\
\hline $\begin{array}{l}23 \text { Octobre } \\
23 \text { Octobre } \\
23 \text { Octobre }\end{array}$ & $\begin{array}{r}8 \mathrm{~h} \\
9 \mathrm{~h} \\
10 \mathrm{~h}\end{array}$ & $\begin{array}{l}\text { Auxerre } \\
\text { Dieppe } \\
\text { Rodez }\end{array}$ & $\begin{array}{l}\text { BOURGOGNE } \\
\text { NORMANDIE } \\
\text { MIDI-PYRÉNÉES }\end{array}$ \\
\hline 25 Octobre & $9 \mathrm{~h}$ & Castres & MIDI-PYRÉNÉES \\
\hline 28 Octobre & $9 \mathrm{~h}$ & Compiègne & PICARDIE \\
\hline $\begin{array}{l}30 \text { Octobre } \\
30 \text { Octobre }\end{array}$ & $\begin{array}{r}9 \text { h } 30 \\
14 \text { h } 15\end{array}$ & $\begin{array}{l}\text { Lyons-la-Forêt } \\
\text { Mont-de-Marsan }\end{array}$ & $\begin{array}{l}\text { NORMANDIE } \\
\text { AQUITAINE }\end{array}$ \\
\hline
\end{tabular}

